

\title{
The characteristic polynomial of the Adams operators on graded connected Hopf algebras
}

\author{
Marcelo Aguiar and Aaron Lauve \\ Dedicated to the memory of Jean-Louis Loday.
}

The Adams operators $\Psi_{n}$ on a Hopf algebra $H$ are the convolution powers of the identity of $H$. They are also called Hopf powers or Sweedler powers. We study the Adams operators when $H$ is graded connected. The main result is a complete description of the characteristic polynomial — both eigenvalues and their multiplicities - for the action of the operator $\Psi_{n}$ on each homogeneous component of $H$. The eigenvalues are powers of $n$. The multiplicities are independent of $n$, and in fact only depend on the dimension sequence of $H$. These results apply in particular to the antipode of $H$, as the case $n=-1$. We obtain closed forms for the generating function of the sequence of traces of the Adams operators. In the case of the antipode, the generating function bears a particularly simple relationship to the one for the dimension sequence. In the case where $H$ is cofree, we give an alternative description for the characteristic polynomial and the trace of the antipode in terms of certain palindromic words. We discuss parallel results that hold for Hopf monoids in species and for $q$-Hopf algebras.

$\begin{array}{ll}\text { Introduction } & 548 \\ \text { 1. Preliminaries on Hopf algebras } & 549 \\ \text { 2. Characteristic polynomials of the Adams operators } & 554 \\ \text { 3. The trace of the Adams operators } & 560 \\ \text { 4. The case of cofree graded connected Hopf algebras } & 564 \\ \text { 5. Examples } & 570 \\ \text { Appendix A. Hopf monoids in species } & 573 \\ \text { Appendix B. q-Hopf algebras } & 576\end{array}$

Aguiar was supported in part by NSF DMS-1001935. Lauve was supported in part by NSA \#H9823012-1-0286. This work was started at Texas A\&M University and both authors are thankful to this institution.

MSC2010: primary 16T05; secondary 16T30.

Keywords: Adams operator, characteristic operation, convolution power, Hopf power, antipode, trace, graded connected Hopf algebra, Hopf monoid in species, $q$-Hopf algebra, Schur indicator, Eulerian idempotent. 


\section{Introduction}

Let $H$ be a Hopf algebra with antipode $\mathrm{S}: H \rightarrow H$. If $H$ is commutative or cocommutative, then it is well known that $\mathrm{S}$ is an involution $\left(\mathrm{S}^{2}=\mathrm{id}\right)$. In particular, its eigenvalues are \pm 1 . Alternatively, if $H$ is finite-dimensional, then $\mathrm{S}$ has finite even order (and its eigenvalues can be arbitrary even roots of unity). This paper studies the behavior of the antipode when $H$ is graded connected. We prove in Corollary 5 that, in this case, the eigenvalues of $S$ are always \pm 1 , even though $S$ may have infinite order on any homogeneous component of $H$.

It is both natural and convenient to consider a more general family of operators on $H$ : the convolution powers of the identity. These are the Adams operators $\Psi_{n}$, and the antipode is $\Psi_{-1}$. Our main result, Theorem 3, provides a complete description of the characteristic polynomial for the operator $\Psi_{n}$ acting on the $m$-th homogeneous component of $H$. For each scalar $n$ and nonnegative integer $m$, the polynomial is uniquely determined by the dimension sequence of $H$. The eigenvalues are powers of $n$. The multiplicities count samples with replacement by length and weight, with the samples taken from a weighted set that arises, numerically, as the inverse Euler transform of the dimension sequence of $H$, and algebraically, as a basis of the graded Lie algebra of primitive elements of a Hopf algebra canonically associated to $H$.

Corollaries 10 and 13 provide information on the trace of the antipode. The former provides a semicombinatorial description for its values, and the latter the following remarkable expression for the generating function:

$$
\sum_{m \geq 0} \operatorname{trace}\left(\left.\mathrm{S}\right|_{H_{m}}\right) t^{m}=\frac{h\left(t^{2}\right)}{h(t)},
$$

where $h(t)$ is the generating function for the dimension sequence of $H$. We derive in Corollary 17 information about the asymptotic behavior of the sequence of traces.

To put these results into perspective, consider the Hopf algebra of symmetric functions. Calculating the trace of the antipode on suitable linear bases yields a simple proof of the interesting identity (see Section 5.1)

$$
c(m)=e(m)-o(m),
$$

where $c(m)$ denotes the number of self-conjugate partitions of $m$, and $e(m)$ and $o(m)$ denote the number of partitions of $m$ with an even number of even parts, and with an odd number of even parts, respectively. The generating function for the 
trace of the antipode in the preceding paragraph may be seen as an extension of this result to arbitrary graded connected Hopf algebras.

Consider now the Hopf algebra of quasisymmetric functions. Another quick calculation reveals that the trace of the antipode on the $m$-th component is a signed count of palindromic compositions of $m$; see Section 5.3. Corollary 22 provides a similar result that applies to any graded connected Hopf algebra that, as a graded coalgebra, is cofree.

Some of the main results admit extensions to certain graded connected $q$-Hopf algebras. Among these, we highlight Corollary 34, which describes the trace of the antipode as a polynomial in $q$, and Corollary 37 , which involves appropriate $q$-generating functions and replaces the above relationship between the sequence of antipode traces and the dimension sequence.

The theory of Hopf monoids in species often runs in parallel to the theory of graded connected Hopf algebras. The main results of the paper admit variants that apply in this context. The sequence of antipode traces is now determined from the dimension sequence as follows (Corollary 29): the exponential generating function of the former is the reciprocal of that of the latter.

The paper is organized as follows. In Section 1, we discuss the necessary preliminaries from Hopf algebra theory. The proof of Theorem 3 is carried out in Section 2. Section 3 focuses on the trace of the Adams operators and the antipode particularly. In Section 4, we give alternatives to our main results about the antipode that hold in the presence of cofreeness assumptions. Section 5 provides illustrations of the results and some simple calculations. In Appendix A we present the results for Hopf monoids in species, and in Appendix B we treat the case of $q$-Hopf algebras.

The present paper supersedes and considerably expands on the results of our extended abstract [Aguiar and Lauve 2013].

\section{Preliminaries on Hopf algebras}

Throughout, all vector spaces are over a field $k$ of characteristic zero.

The structure maps of a bialgebra $H$ are denoted by

$$
\begin{aligned}
\mu: H \otimes H & \rightarrow H, \quad \Delta: H \rightarrow H \otimes H, \\
\iota: \mathbb{k} & \rightarrow H, \quad \varepsilon: H \rightarrow \mathbb{k} .
\end{aligned}
$$

The antipode of a Hopf algebra $H$ is denoted by

$$
\mathrm{S}: H \rightarrow H \text {. }
$$

1.1. Convolution. Let $H$ be a bialgebra and let $\operatorname{End}(H)$ denote the space of linear maps $T: H \rightarrow H$. The convolution product of $P, Q \in \operatorname{End}(H)$ is

$$
P * Q:=\mu \circ(P \otimes Q) \circ \Delta .
$$


This turns the space $\operatorname{End}(H)$ into an associative algebra. The unit element is $\iota \circ \varepsilon$

The bialgebra $H$ is a Hopf algebra if and only if the identity of $H$ is convolutioninvertible. In this case, the antipode $\mathrm{S}$ is the convolution-inverse of the identity map:

$$
\mathrm{S} * \mathrm{id}=\mathrm{id} * \mathrm{~S}=\iota \circ \varepsilon .
$$

Let $H$ be a bialgebra. Put $\Delta^{(0)}:=\mathrm{id}, \Delta^{(1)}:=\Delta$, and

$$
\Delta^{(n)}:=\left(\Delta \otimes \mathrm{id}^{\otimes(n-1)}\right) \circ \Delta^{(n-1)} \text { for all } n \geq 2 .
$$

The superscript is one less than the number of tensor factors in the codomain. Similarly, $\mu^{(n)}$ denotes the map that multiplies $n+1$ elements of $H$, with $\mu^{(0)}:=$ id. The convolution powers of any $T \in \operatorname{End}(H)$ can be written as

$$
T^{* 0}=\iota \circ \varepsilon \quad \text { and } \quad T^{* n}=\mu^{(n-1)} \circ T^{\otimes n} \circ \Delta^{(n-1)} \quad \text { for } n \geq 1 .
$$

1.2. Adams operators. Let $H$ be a Hopf algebra. The convolution powers $i^{* n}$ of the identity of $H$ are defined for any integer $n$. They are called Adams operators and are denoted by

$$
\Psi_{n}:=\mathrm{id}^{* n}: H \rightarrow H
$$

For $n \geq 1$, we have

$$
\Psi_{n}=\mu^{(n-1)} \circ \Delta^{(n-1)} .
$$

Note that $\Psi_{0}=\iota \circ \varepsilon$ and $\Psi_{-1}=$ S. Also,

$$
\Psi_{-n}=\mathrm{S}^{* n}
$$

for all $n$.

This terminology is used in [Cartier 2007, §3.8] and [Loday 1992, §4.5]. Other common terminology for these operators are Hopf powers [ $\mathrm{Ng}$ and Schauenburg 2008], Sweedler powers [Kashina et al. 2006; 2012], and characteristic operations [Gerstenhaber and Schack 1991; Patras 1993]. The paper [Aguiar and Mahajan 2013, §13] studies analogous operators in the context of Hopf monoids in species.

The main goal of this paper is to analyze the characteristic polynomial of these operators when the Hopf algebra $H$ is graded connected.

1.3. Coradical filtration and primitive elements. For more details on the notions reviewed in this section, see [Montgomery 1993, Chapter 5] or [Radford 2012, Chapter 4].

Let $H^{(0)}$ denote the coradical of a bialgebra $H$, and let

$$
H^{(0)} \subseteq H^{(1)} \subseteq H^{(2)} \subseteq \cdots \subseteq H
$$


denote its coradical filtration. We have

$$
H=\bigcup_{m \geq 0} H^{(m)} .
$$

We say that $H$ is connected if $H^{(0)}$ is spanned by the unit element $1 \in H$. In this case, $H$ is a Hopf algebra; see Section 1.4. In addition, $H^{(1)}=H^{(0)} \oplus \mathcal{P}(H)$, where

$$
\mathcal{P}(H):=\{x \in H \mid \Delta(x)=1 \otimes x+x \otimes 1\}
$$

is the space of primitive elements of $H$. More generally, setting $H_{+}:=\operatorname{Ker}(\varepsilon)$ and defining $\Delta_{+}: H_{+} \rightarrow H_{+} \otimes H_{+}$by

$$
\Delta_{+}(x):=\Delta(x)-1 \otimes x-x \otimes 1,
$$

we have $H^{(m)}=H^{(0)} \oplus \operatorname{Ker}\left(\Delta_{+}^{(m)}\right)$, where the iterates of $\Delta_{+}$are defined as for $\Delta$.

Let $H$ be a bialgebra. Let gr $H$ denote the graded vector space associated to the coradical filtration of $H$ :

$$
\text { gr } H:=H^{(0)} \oplus\left(H^{(1)} / H^{(0)}\right) \oplus\left(H^{(2)} / H^{(1)}\right) \oplus\left(H^{(3)} / H^{(2)}\right) \oplus \cdots .
$$

A filtration-preserving map $f: H \rightarrow K$ induces a map

$$
\text { gr } f: \operatorname{gr} H \rightarrow \operatorname{gr} K \text {. }
$$

The structure maps of $H$ are filtration-preserving (when $H \otimes H$ is endowed with the tensor product of the coradical filtrations of each factor). The induced maps turn gr $H$ into a bialgebra. If $H$ is a Hopf algebra, so is gr $H$. If $H$ is connected, so is gr $H$. More importantly:

Lemma 1. If the bialgebra $H$ is connected, then the bialgebra gr $H$ is commutative.

This is an immediate consequence of [Sweedler 1969, Theorem 11.2.5.a]. A direct proof is given in [Aguiar and Sottile 2005a, Proposition 1.6]. We are thankful to Akira Masuoka, Susan Montgomery and the referee for pointing out the reference to Sweedler's text.

The passage $H \mapsto \operatorname{gr} H$ is functorial with respect to filtration-preserving maps. It follows that convolution products are preserved: if $f$ and $g: H \rightarrow H$ are linear maps, then

$$
\operatorname{gr}(f * g)=(\operatorname{gr} f) *(\operatorname{gr} g) .
$$

A morphism of bialgebras $f: H \rightarrow K$ preserves the coradical filtrations. The induced map gr $f: \operatorname{gr} H \rightarrow \operatorname{gr} K$ is a morphism of bialgebras. 
1.4. Antipode and Eulerian idempotents. Any connected bialgebra is a Hopf algebra with antipode

$$
\mathrm{S}=\sum_{k \geq 0}(\iota \circ \varepsilon-\mathrm{id})^{* k}
$$

This basic result can be traced back to Sweedler [1969, Lemma 9.2.3] and Takeuchi [1971, Lemma 14]; see also [Montgomery 1993, Lemma 5.2.10] and [Radford 2012, Lemma 7.6.2]. It follows by expanding

$$
x^{-1}=\frac{1}{1-(1-x)}=\sum_{k \geq 0}(1-x)^{k}
$$

in the convolution algebra, with $x=\mathrm{id}$ and $1=\imath \circ \varepsilon$. Connectedness guarantees that the sum in (6) is finite when evaluated on any $h \in H$. More precisely, if $h \in H^{(m)}$, then $(\mathrm{id}-\iota \circ \varepsilon)^{* k}(h)=0$ for all $k>m$.

Assume for the remainder of the section that $H$ is a connected Hopf algebra.

The binomial theorem yields the following expression for the $n$-th Adams operator for all integers $n$ :

$$
\Psi_{n}=\sum_{k \geq 0}\left(\begin{array}{l}
n \\
k
\end{array}\right)(\mathrm{id}-\iota \circ \varepsilon)^{* k} .
$$

Moreover, the right-hand side of (7) is well-defined for all scalar values of $n$. In this manner, the Adams operators $\Psi_{n}$ are defined for all scalars $n$.

Similarly, the following series expansion defines an element $\log (\mathrm{id})$ in the convolution algebra:

$$
\log (\mathrm{id}):=-\sum_{k \geq 1} \frac{1}{k}(\iota \circ \varepsilon-\mathrm{id})^{* k}
$$

Additionally, consider the elements $\mathrm{e}^{(k)}$, for $k \geq 0$, given by

$$
\mathrm{e}^{(0)}:=\iota \circ \varepsilon, \quad \mathrm{e}^{(1)}:=\log (\mathrm{id}), \quad \mathrm{e}^{(k)}:=\frac{1}{k !}\left(\mathrm{e}^{(1)}\right)^{* k} \quad \text { for } k>1 .
$$

In the case where $H$ is commutative or cocommutative, the $\mathrm{e}^{(k)}$ form a complete orthogonal system of idempotent operators on $H$. That is,

$$
\mathrm{id}=\sum_{k \geq 0} \mathrm{e}^{(k)}, \quad \mathrm{e}^{(k)} \circ \mathrm{e}^{(k)}=\mathrm{e}^{(k)}, \quad \mathrm{e}^{(j)} \circ \mathrm{e}^{(k)}=0 \quad \text { for } j \neq k .
$$

The $\mathrm{e}^{(k)}$ are the higher Eulerian idempotents; $\mathrm{e}^{(1)}$ is the first Eulerian idempotent. It follows from (1), (9), and the identity $x^{* n}=\exp (n \log x)$ that

$$
\Psi_{n}=\sum_{k \geq 0} n^{k} \mathrm{e}^{(k)}
$$


for all scalars $n$. In particular,

$$
\mathrm{S}=\sum_{k \geq 0}(-1)^{k} \mathrm{e}^{(k)}
$$

If $H$ is cocommutative, $\mathrm{e}^{(k)}$ projects onto the subspace spanned by $k$-fold products of primitive elements of $H$. In particular, $\mathrm{e}^{(1)}$ projects onto $\mathcal{P}(H)$.

For proofs of these results, see [Loday 1992, Chapter 4], [Patras 1993] or [Schmitt 1994, §9]. Some instances of these operators in the recent literature include [Diaconis et al. 2014; Novelli et al. 2013; Pang 2014; Patras and Schocker 2006]. For references to earlier work on Eulerian idempotents, see [Aguiar and Mahajan 2013, §14].

1.5. Hopf-Lie theory. Let $H$ be a bialgebra. The space $\mathcal{P}(H)$ is a Lie subalgebra of $H$ under the commutator bracket. Write $\mathfrak{g}$ for the Lie algebra $\mathcal{P}(H)$. If $H$ is connected and cocommutative, the Cartier-Milnor-Moore (CMM) theorem yields a canonical isomorphism of Hopf algebras

$$
H \cong \mathcal{U}(\mathfrak{g})
$$

between $H$ and the enveloping algebra of $\mathfrak{g}$.

Let $\mathcal{S}(V)$ denote the symmetric algebra on a space $V$. It carries a unique Hopf algebra structure for which the elements of $V$ are primitive. The Poincaré-BirkhoffWitt (PBW) theorem furnishes canonical isomorphisms

$$
\mathcal{U}(\mathfrak{g}) \cong \mathcal{S}(\mathfrak{g}) \quad \text { and } \quad \operatorname{gr} \mathcal{U}(\mathfrak{g}) \cong \mathcal{S}(\mathfrak{g})
$$

where $\mathfrak{g}$ is an arbitrary Lie algebra. The former is an isomorphism of coalgebras, the latter of Hopf algebras. Here $\mathcal{S}(\mathfrak{g})$ is the symmetric (Hopf) algebra on the vector space underlying $\mathfrak{g}$.

Proofs of these classical results can be found in [Cartier 2007, §3.8], [Milnor and Moore 1965, § 7] and [Quillen 1969, Appendix B]. For additional references, see [Kassel 1995, Theorem V.2.5], [Loday 1992, §3.3.4 and Appendix A], and [Montgomery 1993, Theorem 5.6.5].

Lemma 1 provides a construction of a commutative connected Hopf algebra gr $H$ from an arbitrary connected Hopf algebra $H$. This will enable us to employ CMM and PBW in a wider setting than that of (co)commutative Hopf algebras.

1.6. Graded bialgebras. The bialgebra $H$ is graded if there is given a vector space decomposition

$$
H=\bigoplus_{m \geq 0} H_{m}
$$


such that

$$
\begin{gathered}
\mu\left(H_{p} \otimes H_{q}\right) \subseteq H_{p+q} \quad \text { for all } p, q \geq 0, \\
\Delta\left(H_{m}\right) \subseteq \bigoplus_{p+q=m} H_{p} \otimes H_{q} \quad \text { for all } m \geq 0, \\
\operatorname{Im}(\iota) \subseteq H_{0}, \quad \text { and } \quad H_{m} \subseteq \operatorname{Ker}(\varepsilon) \quad \text { for all } m>0 .
\end{gathered}
$$

(The condition on the unit map $\iota$ simply states that $1 \in H_{0}$.) If $H$ is Hopf and the antipode satisfies

$$
\mathrm{S}\left(H_{m}\right) \subseteq H_{m}
$$

for all $m \geq 0$, we say that $H$ is a graded Hopf algebra.

If $H$ is an arbitrary bialgebra, then gr $H$ is a graded bialgebra for which the component of degree $n$ is $H^{(n)} / H^{(n-1)}$.

Let $H$ be a graded bialgebra. The space $\mathcal{P}(H)$ is then graded with $\mathcal{P}(H)_{m}=$ $\mathcal{P}(H) \cap H_{m}$. Moreover, $\mathcal{P}(H)$ is then a graded Lie algebra. Similarly, each subspace $H^{(n)}$ is graded with $\left(H^{(n)}\right)_{m}=H^{(n)} \cap H_{m}$. Hence, gr $H$ inherits a second grading for which

$$
(\operatorname{gr} H)_{m}:=\left(H^{(0)}\right)_{m} \oplus\left(\left(H^{(1)}\right)_{m} /\left(H^{(0)}\right)_{m}\right) \oplus\left(\left(H^{(2)}\right)_{m} /\left(H^{(1)}\right)_{m}\right) \cdots .
$$

Moreover, $H^{(0)} \subseteq H_{0}$.

We say that $H$ is graded connected if $\operatorname{dim} H_{0}=1$. The preceding implies that in this case $H_{0}=H^{(0)}$, and therefore $H$ is indeed connected.

Assume that $H$ is a graded connected bialgebra. One may show by induction that $H_{m} \subseteq H^{(m)}$ for all $m$. It follows that the sum (12) stops at $\left(H^{(m)}\right)_{m} /\left(H^{(m-1)}\right)_{m}$. It also follows, from (6), that $H$ is a graded Hopf algebra and

$$
\left.\mathrm{S}\right|_{H_{m}}=\left.\sum_{k=0}^{m}(\iota \circ \varepsilon-\mathrm{id})^{* k}\right|_{H_{m}} .
$$

More generally, it follows from (7) that

$$
\left.\Psi_{n}\right|_{H_{m}}=\left.\sum_{k=0}^{m}\left(\begin{array}{l}
n \\
k
\end{array}\right)(\mathrm{id}-\iota \circ \varepsilon)^{* k}\right|_{H_{m}} .
$$

\section{Characteristic polynomials of the Adams operators}

This section contains the main result (Theorem 3), which determines the characteristic polynomials of the Adams operators on a graded connected Hopf algebra $H$. These only depend on the dimension sequence of $H$. A number of consequences about the antipode are also presented. Some preliminaries on enumeration of multisets are reviewed first. 
2.1. Sampling with replacement and the symmetric algebra. Given a sequence $\boldsymbol{g}=\left(g_{i}\right)_{i \geq 1}$ and a partition $\lambda=1^{k_{1}} 2^{k_{2}} \ldots r^{k_{r}}$, put

$$
\left(\begin{array}{l}
g \\
\lambda
\end{array}\right):=\left(\begin{array}{c}
g_{1}+k_{1}-1 \\
k_{1}
\end{array}\right) \cdots\left(\begin{array}{c}
g_{r}+k_{r}-1 \\
k_{r}
\end{array}\right) .
$$

Let $|\lambda|:=k_{1}+2 k_{2}+\cdots+r k_{r}$ denote the size of $\lambda$, and $\ell(\lambda):=k_{1}+k_{2}+\cdots+k_{r}$ denote the number of parts of $\lambda$. Given nonnegative integers $k$ and $m$, set

In particular,

$$
\operatorname{mul}(k, m):=\sum_{\substack{|\lambda|=m \\
\ell(\lambda)=k}}\left(\begin{array}{l}
\boldsymbol{g} \\
\lambda
\end{array}\right) .
$$

$$
\operatorname{mul}(0, m)=\delta(0, m) \quad \text { and } \quad \operatorname{mul}(k, m)=0 \quad \text { for all } k>m .
$$

The numbers $\operatorname{mul}(k, m)$ depend on the given sequence, although this is not reflected in the notation.

If we sample with replacement from a set with $g_{i}$ elements of weight $i$, then $\left(\begin{array}{l}g \\ \lambda\end{array}\right)$ counts the number of samples with weight distribution $\lambda$; in other words, the number of multisets of cardinality $\ell(\lambda)$ containing exactly $k_{i}$ elements of weight $i$ for each $i=1, \ldots, r$. The numbers $\operatorname{mul}(k, m)$ then count the number of multisets of cardinality $k$ and total weight $m$.

Now let $W$ be a positively graded vector space, and let $g_{i}:=\operatorname{dim} W_{i}$ for each $i \geq 1$. Let $W^{k}$ denote the $k$-th symmetric power of $W$. In other words, $W^{k}$ is the subspace of the symmetric algebra $\mathcal{S}(W)$ spanned by $k$-fold products of elements of $W$. It inherits a grading from $W$, where $\left(W^{k}\right)_{m}$ is spanned by products $w_{1} \cdots w_{k}$ with $\operatorname{deg}\left(w_{1}\right)+\cdots+\operatorname{deg}\left(w_{k}\right)=m$.

Fix a homogeneous basis of $W$, and let it be our sample set. The set of monomials of length $k$ (that is, $k$-fold products of basis elements of $W$ ) is then a basis of $W^{k}$. A multiset of cardinality $k$ and total weight $m$ corresponds to a monomial of length $k$ and degree $m$. Therefore,

$$
\operatorname{dim}\left(W^{k}\right)_{m}=\operatorname{mul}(k, m) .
$$

The bivariate generating series for $\mathcal{S}(W)$ by length and degree is then

$$
\sum_{k, m \geq 0} \operatorname{mul}(k, m) s^{k} t^{m}=\prod_{i \geq 1}\left(1-s t^{i}\right)^{-g_{i}}
$$

This follows by expanding the right-hand side with the aid of the binomial theorem and employing (15) and (16).

The numbers $\operatorname{mul}(k, m)$ enter in Theorem 3 below. 
2.2. Characteristic polynomial. We state some standard results from linear algebra.

Lemma 2. Let $V$ be a finite-dimensional vector space and $T \in \operatorname{End}(V)$ a linear transformation.

(i) Let $U$ be a $T$-invariant subspace of $V$. If $T^{\uparrow} \in \operatorname{End}(V / U)$ denotes the linear transformation induced by $T$ on the quotient, and $T_{\downarrow} \in \operatorname{End}(U)$ denotes the restriction of $T$ to $U$, then the characteristic polynomials of these three maps satisfy

$$
\chi_{T}(x)=\chi_{T_{\downarrow}}(x) \chi_{T \uparrow}(x) .
$$

(ii) The characteristic polynomials of $T$ and of the dual map $T^{*} \in \operatorname{End}\left(V^{*}\right)$ are equal.

We are now ready for our main result. Let $H$ be a graded connected Hopf algebra. We assume from this point onwards that the homogeneous components $H_{m}$ of $H$ are finite-dimensional. We let $\tilde{H}$ denote the graded dual of gr $H$ with respect to the grading (12). It is a graded bialgebra with homogeneous components

$$
\tilde{H}_{m}:=\left((\operatorname{gr} H)_{m}\right)^{*} .
$$

If $H$ is graded connected, $\widetilde{H}$ is graded connected and cocommutative, by Lemma 1 . We let $\mathfrak{g}:=\mathcal{P}(\tilde{H})$ denote the graded Lie algebra of primitive elements of $\tilde{H}$. For each $i \geq 1$, let

$$
g_{i}:=\operatorname{dim} \mathfrak{g}_{i}
$$

denote the dimension of the homogeneous component of $\mathfrak{g}$ of degree $i$. Consider the corresponding numbers $\operatorname{mul}(k, m)$, as in (16).

Theorem 3. Let $H$ be as above. For every scalar $n$ and nonnegative integer $m$, the characteristic polynomial of the restriction $\left.\Psi_{n}\right|_{H_{m}}$ of the $n$-th Adams operator is

$$
\chi\left(\left.\Psi_{n}\right|_{H_{m}}\right)(x)=\prod_{k=0}^{m}\left(x-n^{k}\right)^{\mathrm{mul}(k, m)} .
$$

Before the proof, a few remarks are in order. First, note that the factor indexed by $k=0$ is nontrivial only when $m=0$, according to (17). Second, note that the exponents $\operatorname{mul}(k, m)$ do not depend on $n$. Additional information on the exponents $\operatorname{mul}(k, m)$ is provided in Proposition 4.

Proof. First of all, it suffices to establish (19) when $n$ is a nonnegative integer. Indeed, both sides depend polynomially on $n$ - the left-hand side in view of (14).

We argue that we may replace $H$ with gr $H$. Indeed, since $\Psi_{n}$ preserves both the grading and the coradical filtration of $H$, it preserves the filtration

$$
\left(H^{(0)}\right)_{m} \subseteq\left(H^{(1)}\right)_{m} \subseteq \cdots \subseteq\left(H^{(m)}\right)_{m}=H_{m}
$$


for each $m$. By repeated application of Lemma 2(i), we deduce that

$$
\chi\left(\left.\Psi_{n}\right|_{H_{m}}\right)=\chi\left(\left.\operatorname{gr}\left(\Psi_{n}\right)\right|_{(\operatorname{gr} H)_{m}}\right) .
$$

In addition, by (5),

$$
\operatorname{gr}\left(\Psi_{n}\right)=\operatorname{gr}\left(\mathrm{id}^{* n}\right)=(\operatorname{grid})^{* n}=\mathrm{id}^{* n}=\Psi_{n} .
$$

Therefore,

$$
\chi\left(\left.\Psi_{n}\right|_{H_{m}}\right)=\chi\left(\left.\Psi_{n}\right|_{(\mathrm{gr} H)_{m}}\right) .
$$

Next, we may replace gr $H$ with $\widetilde{H}$. Indeed, the map $T \mapsto T^{*}$ is an isomorphism of convolution algebras $\operatorname{End}(H) \cong \operatorname{End}\left(H^{*}\right)$ (where duals and endomorphisms are in the graded sense). Together with Lemma 2(ii) this implies that

$$
\chi\left(\left.\Psi_{n}\right|_{(\text {gr } H)_{m}}\right)=\chi\left(\left.\Psi_{n}\right|_{\widetilde{H}_{m}}\right) .
$$

Now let $\mathfrak{g}=\mathcal{P}(\widetilde{H})$. By CMM, $\widetilde{H} \cong \mathcal{U}(\mathfrak{g})$, and by PBW, $\operatorname{gr} \mathcal{U}(\mathfrak{g}) \cong \mathcal{S}(\mathfrak{g})$ as Hopf algebras. The same argument as above shows that we may replace $\tilde{H}$ with $\mathcal{S}(\mathfrak{g})$.

As $\mathcal{S}(\mathfrak{g})$ is cocommutative, the Eulerian idempotents are available. From (11) we have that

$$
\chi\left(\left.\Psi_{n}\right|_{\mathcal{S}(\mathfrak{g})_{m}}\right)=\prod_{k \geq 0} \chi\left(\left.n^{k} \mathrm{e}^{(k)}\right|_{\mathcal{S}(\mathfrak{g})_{m}}\right) .
$$

It thus suffices to calculate the characteristic polynomial of the $e^{(k)}$ on $\mathcal{S}(\mathfrak{g})$.

Finally, the action of $\mathrm{e}^{(k)}$ on $\mathcal{S}(\mathfrak{g})$ is simply a projection onto $\mathfrak{g}^{k}$, the subspace spanned by $k$-fold products of elements of $\mathfrak{g}$. It follows that

$$
\chi\left(\left.n^{k} \mathrm{e}^{(k)}\right|_{\mathcal{S}(\mathfrak{g})_{m}}\right)(x)=\left(x-n^{k}\right)^{\mathrm{mul}(k, m)},
$$

where

$$
\operatorname{mul}(k, m)=\operatorname{dim}\left(\mathfrak{g}^{k}\right)_{m}
$$

This completes the proof.

Remark. One may easily see that the Adams operators act on $\mathcal{S}(W)$ as follows:

$$
\Psi_{n}\left(w_{1} \cdots w_{k}\right)=n^{k} w_{1} \cdots w_{k},
$$

where $w_{i} \in W, i=1, \ldots, k$. The proof of Theorem 3 can then be completed without explicit mention of the Eulerian idempotents.

On the other hand, assume that $H$ is a graded connected Hopf algebra that is either commutative or cocommutative. The expression (11) for the Adams operators in terms of the Eulerian idempotents shows that the former are simultaneously diagonalizable. The thesis of Amy Pang [2014] contains a discussion of a common eigenbasis for the Adams operators on such $H$. 
The exponents $\operatorname{mul}(k, m)$ are determined by the dimension sequence of $\mathfrak{g}$, through (15) and (16). In turn, this sequence is related to the dimension sequence of $H$ by

$$
1+\sum_{m \geq 1} h_{m} t^{m}=\prod_{i \geq 1}\left(1-t^{i}\right)^{-g_{i}},
$$

where $h_{m}:=\operatorname{dim} H_{m}$. Indeed, the right-hand side is the generating series for $\mathcal{S}(\mathfrak{g})$, and we have from the proof of Theorem 3 that $H_{m} \cong \widetilde{H}_{m} \cong \mathcal{S}(\mathfrak{g})_{m}$ as vector spaces. It follows that the sequences $\left(g_{i}\right)$ and $\left(h_{m}\right)$ determine each other. In particular:

Proposition 4. The exponents $\mathrm{mul}(k, m)$ are determined by the dimension sequence of $H$.

Remark. Equation (20) says that the sequence $\left(h_{m}\right)_{m \geq 1}$ is the Euler transform of $\left(-g_{i}\right)_{i \geq 1}$, in the sense of [Sloane and Plouffe 1995, p. 20]. The nonnegativity of the sequence $\left(g_{i}\right)$ restricts the class of sequences $\left(h_{m}\right)$ that may be realized as dimension sequences of graded connected Hopf algebras.

2.3. Eigenvalues of the antipode. Let $H$ be a graded connected Hopf algebra and $\mathrm{S}$ its antipode.

Applying Theorem 3 in the case $n=-1$ yields information about the antipode, since $\mathrm{S}=\Psi_{-1}$. We obtain

$$
\chi\left(\left.\mathrm{S}\right|_{H_{m}}\right)(x)=(x-1)^{\mathrm{emul}(m)}(x+1)^{\mathrm{omul}(m)},
$$

where

$$
\operatorname{emul}(m):=\sum_{k \text { even }} \operatorname{mul}(k, m) \quad \text { and } \operatorname{omul}(m):=\sum_{k \text { odd }} \operatorname{mul}(k, m) .
$$

In particular:

Corollary 5. The eigenvalues of the antipode are \pm 1 .

Remark. Corollary 5 fails for general Hopf algebras. Let $\omega$ be a primitive cube root of unity and consider Taft's Hopf algebra $T_{3}(\omega)$ [Taft 1971], with generators $\{g, x\}$ and relations $\left\{g^{3}=1, x^{3}=0, g x=\omega x g\right\}$. The coproduct and antipode are determined by $\Delta(g)=g \otimes g, \mathrm{~S}(g)=g^{-1}, \Delta(x)=1 \otimes x+x \otimes g$, and $\mathrm{S}(x)=-x g^{-1}$. Here $x^{2}+\omega x^{2} g$ is an eigenvector of $\mathrm{S}$ with eigenvalue $\omega$.

From Corollary 5 we deduce:

Corollary 6. The antipode is diagonalizable if and only if it is an involution.

Remark. The antipode of a graded connected Hopf algebra need not be an involution (or equivalently, diagonalizable). For example, consider the MalvenutoReutenauer Hopf algebra of permutations (Example 8). Its antipode is of infinite order already on the homogeneous component of degree 3; see Remark 5.6 in [Aguiar and Sottile 2005b]. 
2.4. Composition powers of the antipode. Consider $S^{2}$, the composition of the antipode $S$ with itself. Corollary 5 implies that 1 is the only eigenvalue of $S^{2}$, so $\mathrm{S}^{2}-\mathrm{id}$ is nilpotent on each homogeneous component. We have a more precise result.

Proposition 7. The map $\left.\left(\mathrm{S}^{2}-\mathrm{id}\right)\right|_{H_{m}}$ is nilpotent of order at most $m$.

Proof. Since $\operatorname{gr}(H)$ is commutative, $\mathrm{S}^{2}=\mathrm{id}$ on $\operatorname{gr}(H)$. Hence $\left(\mathrm{S}^{2}-\mathrm{id}\right)\left(H^{(m)}\right) \subseteq$ $H^{(m-1)}$ for all $m \geq 1$. On $H^{(1)}=\mathbb{k} \oplus \mathcal{P}(H)$, we have $\mathrm{S}= \pm \mathrm{id}$, and then $\mathrm{S}^{2}-\mathrm{id}=0$. By induction, $\left(\mathrm{S}^{2}-\mathrm{id}\right)^{m}\left(H^{(m)}\right)=0$ for all $m \geq 1$. The statement follows by recalling that $H_{m} \subseteq H^{(m)}$.

Remark. Let $d_{m}$ be the order of nilpotency of $\left.\left(\mathrm{S}^{2}-\mathrm{id}\right)\right|_{H_{m}}$, so that $1 \leq d_{m} \leq m$ by Proposition 7 . The lower bound $d_{m}=1$ is attained by any involutory Hopf algebra for all $m$. Computations suggest that the Hopf algebra of signed permutations [Bonnafé and Hohlweg 2006] attains the upper bound $d_{m}=m$ for all $m \geq 1$.

Example 8. Consider the Hopf algebra $H=\mathfrak{S S y m}$ introduced by Malvenuto and Reutenauer [1995]. We claim that $d_{m} \leq m-1$ for $m \geq 2$ (and $d_{1}=1$ ). Let $\operatorname{id}_{m}=123 \cdots m$ be the identity permutation, in one-line notation, and let $\omega_{m}$ be the longest permutation of $m$ elements, $\omega_{m}=m \cdots 321$. Let $\mathcal{F}$ and $\mathcal{M}$ denote the fundamental and monomial bases of $H$, in the notation of [Aguiar and Sottile 2005b]. It follows from Corollary 6.3 of the same paper that

$$
H_{m}=\left(H_{m} \cap H^{(m-1)}\right) \oplus K_{m},
$$

where $K_{m}$ is the one-dimensional space spanned by $\mathcal{F}_{\omega_{m}}=\mathcal{M}_{\omega_{m}}$. The proof of Proposition 7 shows that on $H_{m} \cap H^{(m-1)}$ the order of nilpotency is at most $m-1$. On the other hand, it is easy to see that

$$
\mathrm{S}\left(\mathcal{F}_{\omega_{m}}\right)=(-1)^{m} \mathcal{F}_{\mathrm{id}_{m}} \text { and } \mathrm{S}\left(\mathcal{F}_{\mathrm{id}_{m}}\right)=(-1)^{m} \mathcal{F}_{\omega_{m}}
$$

Therefore, $\mathrm{S}^{2}$ is the identity on $K_{m}$. The claim follows.

We turn to higher composition powers of the antipode. Since $H^{\text {op }}$ is another graded connected Hopf algebra, it possesses an antipode, and this is the inverse of S by [Montgomery 1993, Lemma 1.5.11] or [Aguiar and Mahajan 2013, Proposition 1.23]. In particular, $S$ is invertible, and we may consider powers $S^{n}$ for any integer $n$.

Proposition 9. For any integer $n$,

$$
\chi\left(\left.\mathrm{S}^{n}\right|_{H_{m}}\right)(x)= \begin{cases}\chi\left(\left.\mathrm{id}\right|_{H_{m}}\right)(x) & \text { if } n \text { is even, } \\ \chi\left(\left.\mathrm{S}\right|_{H_{m}}\right)(x) & \text { if } n \text { is odd } .\end{cases}
$$

Proof. As in the proof of Theorem 3, we may assume that $H$ is commutative. In this case, $\mathrm{S}^{2}=\mathrm{id}$ and hence $\mathrm{S}^{n}=\mathrm{id}$ for even $n$ and $\mathrm{S}^{n}=\mathrm{S}$ for odd $n$. 


\section{The trace of the Adams operators}

We study the trace of the Adams operators on $H$. The generating functions for their sequences of traces admit closed expressions in terms of the inverse Euler transform of the dimension sequence of $H$. The generating function for the trace of the antipode is particularly remarkable.

3.1. Generating functions for the trace. We return to the situation of Theorem 3. Thus, $H$ is a graded connected Hopf algebra, and the integers mul $(k, m)$ are determined by its dimension sequence through (15), (16), and (20).

As an immediate consequence of this theorem, we have:

Corollary 10. For all scalars $n$ and nonnegative integers $m$,

$$
\operatorname{trace}\left(\left.\Psi_{n}\right|_{H_{m}}\right)=\sum_{k=0}^{m} n^{k} \operatorname{mul}(k, m) .
$$

In particular,

$$
\operatorname{trace}\left(\left.\mathrm{S}\right|_{H_{m}}\right)=\sum_{k=0}^{m}(-1)^{k} \operatorname{mul}(k, m)=\operatorname{emul}(m)-\operatorname{omul}(m) .
$$

We turn to generating functions for the trace. First we consider the $n$-th Adams operator. Recall that the sequence $\left(g_{i}\right)$ is determined by the dimension sequence of $H$ through (20).

Corollary 11. For all scalars $n$,

$$
\sum_{m \geq 0} \operatorname{trace}\left(\left.\Psi_{n}\right|_{H_{m}}\right) t^{m}=\prod_{i \geq 1}\left(1-n t^{i}\right)^{-g_{i}} .
$$

Proof. This follows at once from (18) and (23).

For each scalar $n$, let

$$
h_{n}(t):=\sum_{m \geq 0} \operatorname{trace}\left(\left.\Psi_{n}\right|_{H_{m}}\right) t^{m}
$$

denote the generating function for the sequence of traces of $\Psi_{n}$. As a consequence of Corollary 11, these functions satisfy certain interesting relations. In order to state them, let

$$
\mu_{k}:=\left\{\omega \in \mathbb{C} \mid \omega^{k}=1\right\}
$$

denote the group of complex $k$-th roots of unity.

Proposition 12. For each scalar $n$ and positive integer $k$,

$$
h_{n^{k}}\left(t^{k}\right)=\prod_{\omega \in \mu_{k}} h_{\omega n}(t) .
$$


In particular,

$$
h_{n^{2}}\left(t^{2}\right)=h_{n}(t) h_{-n}(t) .
$$

Proof. We employ the factorization

$$
1-t^{k}=\prod_{\omega \in \mu_{k}}(1-\omega t) .
$$

Then, from (25), we have

$$
h_{n^{k}}\left(t^{k}\right)=\prod_{i \geq 1}\left(1-n^{k} t^{k i}\right)^{-g_{i}}=\prod_{i \geq 1} \prod_{\omega \in \mu_{k}}\left(1-\omega n t^{i}\right)^{-g_{i}}=\prod_{\omega \in \mu_{k}} h_{\omega n}(t) .
$$

The generating function for the trace of the antipode takes a special form. Let

$$
h(t):=1+\sum_{m \geq 1} h_{m} t^{m}
$$

denote the generating function for the dimension sequence of $H$.

Corollary 13.

$$
\sum_{m \geq 0} \operatorname{trace}\left(\left.\mathrm{S}\right|_{H_{m}}\right) t^{m}=\frac{h\left(t^{2}\right)}{h(t)}
$$

Proof. Since $\Psi_{1}=$ id and $\Psi_{-1}=$ S, we have $h(t)=h_{1}(t)$ and

$$
\sum_{m \geq 0} \operatorname{trace}\left(\left.\mathrm{S}\right|_{H_{m}}\right) t^{m}=h_{-1}(t) .
$$

Thus, (28) is the case $n=1$ of (27).

Remark. Corollary 13 shows that the sequence of antipode traces is determined by the dimension sequence in a simple manner. The result also shows that, conversely, the dimension sequence is determined by the sequence of antipode traces since the relation (28) can be solved for $h(t)$.

Example 14. Suppose the dimension sequence of $H$ is given by $h_{m}:=r^{m}$, where $r$ is a fixed nonnegative integer. Then $h(t)=1 /(1-r t)$ and

$$
\frac{h\left(t^{2}\right)}{h(t)}=\frac{1-r t}{1-r t^{2}}=\sum_{n \geq 0} r^{n} t^{2 n}-\sum_{n \geq 0} r^{n+1} t^{2 n+1} .
$$

It follows from (28) that

$$
\operatorname{trace}\left(\left.\mathrm{S}\right|_{H_{m}}\right)= \begin{cases}r^{m / 2} & \text { if } m \text { is even, } \\ -r^{(m+1) / 2} & \text { if } m \text { is odd. }\end{cases}
$$

We have computed the trace of the antipode without knowing anything other than the dimension sequence of $H$. A Hopf algebra with the given dimension sequence is the free algebra on $r$ primitive generators of degree 1; a direct computation of the trace can then be carried out. We do this for the dual Hopf algebra in Example 23. 
We record the generating function for the trace of the composition powers of the antipode.

Corollary 15. For any integer $n$,

$$
\sum_{m \geq 0} \operatorname{trace}\left(\left.\mathrm{S}^{n}\right|_{H_{m}}\right) t^{m}= \begin{cases}h(t) & \text { if } n \text { is even } \\ h\left(t^{2}\right) / h(t) & \text { if } n \text { is odd } .\end{cases}
$$

Proof. This follows from Proposition 9 and Corollary 13.

3.2. The trace of the antipode versus the dimension sequence. We write

$$
a_{m}:=\operatorname{trace}\left(\left.\mathrm{S}\right|_{H_{m}}\right)
$$

for each nonnegative integer $m$. Let $a(t)$ be its generating function.

We analyze the behavior of the sequence $\left(a_{m}\right)$ in relation to the dimension sequence $\left(h_{m}\right)$.

Corollary 16. If the sequence $\left(h_{m}\right)$ satisfies a linear recursion with constant coefficients, then so does $\left(a_{m}\right)$.

Proof. We employ [Stanley 2012, Theorem 4.1.1]. In this situation, the series $h(t)$ is rational, and hence so is $a(t)$ by (28).

We turn to asymptotics. We assume there exists a meromorphic function $h(z)$ of a complex variable $z$, holomorphic on a neighborhood of 0 , and such that its Taylor expansion is the generating function $h(t)$. It then follows from Pringsheim's theorem [Flajolet and Sedgewick 2009, Theorem IV.6] that a dominant singularity occurs at a positive real number $R$. Moreover, $R \leq 1$. Indeed, if $R>1$, the coefficients $h_{m}$ would approach 0 by the exponential growth formula [ibid., Theorem IV.7], and this would force the integers $h_{m}$ to be 0 from a point on. (As we remark at the end of Section 3.3, this can only happen if $H$ is the one-dimensional Hopf algebra, a triviality which we exclude from consideration.)

Corollary 17. Suppose that $R$ is the unique singularity of $h(z)$ in the disk $|z| \leq R^{1 / 4}$. Let $\gamma$ be the order of this singularity. Suppose further that $h(z)$ is nonzero in the disk $|z| \leq R^{1 / 2}$, and

$$
h\left(-R^{1 / 2}\right) \neq \pm h\left(R^{1 / 2}\right) .
$$

Then

$$
\frac{a_{m}}{h_{m}} \sim \frac{R^{m / 2}}{2^{\gamma}}\left(\frac{1}{h\left(R^{1 / 2}\right)}+(-1)^{m} \frac{1}{h\left(-R^{1 / 2}\right)}\right) .
$$

Proof. The hypotheses guarantee that $R$ is the unique dominant singularity for $h(z)$ and also that $\pm R^{1 / 2}$ are the unique dominant singularities for $a(z)=h\left(z^{2}\right) / h(z)$. 
We then have the standard approximations [Flajolet and Sedgewick 2009, §B.IV; Wilf 2006, §5.2]

$$
h_{m} \sim \frac{1}{\Gamma(\gamma)} m^{\gamma-1} R^{-m} h^{*}(R)
$$

and

$$
a_{m} \sim \frac{2^{\gamma}}{\Gamma(\gamma)} m^{\gamma-1} R^{-m / 2} h^{*}(R)\left(\frac{1}{h\left(R^{1 / 2}\right)}+(-1)^{m} \frac{1}{h\left(-R^{1 / 2}\right)}\right),
$$

where $\Gamma$ is the gamma function and $h^{*}(R)=\lim _{z \rightarrow R}(1-z / R)^{\gamma} h(z)$. The result follows.

Example 18. Suppose the dimension sequence is given by

$$
h_{0}=h_{1}=h_{2}:=1 \quad \text { and } \quad h_{m}:=h_{m-1}+h_{m-2} \quad \text { for all } m \geq 3 .
$$

Thus, for $m \geq 1$, the $h_{m}$ are the Fibonacci numbers. In this case,

$$
h(z)=\frac{z^{2}-1}{z^{2}+z-1}=\left(z^{2}-1\right)(z+\phi)^{-1}(z-1 / \phi)^{-1},
$$

where $\phi=(1+\sqrt{5}) / 2$ is the golden ratio. The hypotheses of Corollary 17 are satisfied with $R=1 / \phi$ and $\gamma=1$. We obtain from (30) the approximation

$$
\frac{a_{m}}{h_{m}} \sim \begin{cases}\phi^{-m / 2} & \text { if } m \text { is even, } \\ -\phi^{(-m+3) / 2} & \text { if } m \text { is odd. }\end{cases}
$$

A Hopf algebra with this dimension sequence is discussed in Section 5.4, and the sequence $a_{m}$ is computed explicitly; see (52). The above may then be seen to follow from the well-known approximation $h_{m} \sim \phi^{m} / \sqrt{5}$ for the Fibonacci numbers.

3.3. Schur indicators. A theme occurring in the recent Hopf algebra literature involves a generalization of the Frobenius-Schur indicator function of a finite group. If $\rho: G \rightarrow \operatorname{End}(V)$ is a complex representation of $G$, then the (second) indicator is

$$
\nu_{2}(G, \rho):=\frac{1}{|G|} \sum_{g \in G} \operatorname{trace} \rho\left(g^{2}\right) .
$$

The only values this invariant can take on irreducible representations are $0,1,-1$, and this occurs precisely when $V$ is a complex, real, or quaternionic representation, respectively [Serre 1977, Proposition 39]. In [Linchenko and Montgomery 2000], a reformulation of the definition was given in terms of convolution powers of the integral $^{1}$ in $\mathbb{C} G$. This extended the notion of (higher) Schur-indicators to all finitedimensional Hopf algebras, and has since become a valuable tool for the study of these algebras [Kashina et al. 2002; Ng and Schauenburg 2008; Sage and Vega

\footnotetext{
${ }^{1}$ This construct, present for finite-dimensional Hopf algebras, is unavailable for general graded connected Hopf algebras.
} 
2012; Shimizu 2012]. In the case where $\rho$ is the regular representation (and $H$ is semisimple), it is shown in [Kashina et al. 2006] that the higher Schur indicators can be reformulated further, removing all mention of the integral: for all nonnegative integers $n$,

$$
v_{n+1}(H)=\operatorname{trace}\left(\mathrm{S} \circ \Psi_{n}\right) .
$$

See also [Kashina et al. 2012]. Our results lead to the following formula for these invariants in the case where $H$ is graded connected (instead of finite-dimensional).

Corollary 19. Let $H$ be a graded connected Hopf algebra. Then, for all scalars $n$ and nonnegative integers $m$,

$$
\operatorname{trace}\left(\mathrm{S} \circ \Psi_{n}\right)=\sum_{k=0}^{m}(-n)^{k} \operatorname{mul}(k, m),
$$

where $\operatorname{mul}(k, m)$ is as in Theorem 3.

Proof. As in the proof of Theorem 3, we may assume that $H$ is commutative. Then $\mathrm{S}$ is a morphism of algebras, and, using (2) and (3), we have

$$
\mathrm{S} \circ \Psi_{n}=\mathrm{S} \circ \mu^{(n-1)} \circ \Delta^{(n-1)}=\mu^{(n-1)} \circ \mathrm{S}^{\otimes n} \circ \Delta^{(n-1)}=\mathrm{S}^{* n}=\Psi_{-n} .
$$

The result follows from (23).

Remark. We mention in passing that the only Hopf algebra $H$ that is at the same time connected and finite-dimensional is the (unique) one-dimensional Hopf algebra. Indeed, combining Lemma 1 with CMM and PBW (as in the proof of Theorem 3), we have $H \cong \mathcal{S}(V)$ as vector spaces for some space $V$. But the only space $V$ for which the symmetric algebra is finite-dimensional is $V=0$. Hence $H \cong \mathbb{k}$. The situation is of course different over fields of positive characteristic or for $(-1)$-Hopf algebras.

\section{The case of cofree graded connected Hopf algebras}

We study the characteristic polynomial and the trace of the antipode of a graded connected Hopf algebra that, as a graded coalgebra, is cofree. Since the former are invariant under duality, the results apply as well to graded connected Hopf algebras that are free as algebras. (We make no further mention of this point as we proceed.)

4.1. Cofreeness. A graded connected Hopf algebra $H$ is cofree if, as a graded coalgebra, it is isomorphic to a deconcatenation coalgebra $\mathcal{T}^{\vee}(V)$ on a graded vector space $V$. The underlying space of the latter is

$$
\mathcal{T}^{\vee}(V):=\bigoplus_{k \geq 0} V^{\otimes k}
$$


(the same as that of the tensor algebra $\mathcal{T}(V))$. The coproduct on a $k$-fold tensor is $\Delta\left(x_{1} \cdots x_{k}\right)=1 \otimes\left(x_{1} \cdots x_{k}\right)+\sum_{i=1}^{k-1}\left(x_{1} \cdots x_{i}\right) \otimes\left(x_{i+1} \cdots x_{k}\right)+\left(x_{1} \cdots x_{k}\right) \otimes 1$.

For more details, see [Aguiar and Mahajan 2010, §2.6] or [Radford 2012, §4.5].

In this situation, we have

as graded vector spaces.

$$
H^{(m)} \cong \bigoplus_{k=0}^{m} V^{\otimes k} \quad \text { and } \quad \mathcal{P}(H) \cong V
$$

The shuffle product of two tensors $x_{1} \cdots x_{i}$ and $x_{i+1} \cdots x_{k}$ is the following element of $\mathcal{T}^{\vee}(V)$ :

$$
\sum_{\sigma} x_{\sigma^{-1}(1)} \cdots x_{\sigma^{-1}(k)}
$$

where the sum is over all permutations $\sigma \in S_{k}$ such that

$$
\sigma(1)<\cdots<\sigma(i) \text { and } \sigma(i+1)<\cdots<\sigma(k) .
$$

The shuffle product turns the deconcatenation coalgebra $\mathcal{T}^{\vee}(V)$ into a commutative graded connected Hopf algebra. The antipode acts on a tensor by reversing the components:

$$
\mathrm{S}\left(x_{1} x_{2} \cdots x_{k}\right)=(-1)^{k} x_{k} \cdots x_{2} x_{1} .
$$

Under the assumption of cofreeness, there exists a stronger version of Lemma 1. Lemma 20. Let $H$ be a graded connected Hopf algebra that is cofree as a graded coalgebra. Then

$$
\operatorname{gr} H \cong \mathcal{T}^{\vee}(V)
$$

as graded Hopf algebras, where $V=\mathcal{P}(H)$.

This result appears in [Aguiar and Sottile 2005b, Proposition 1.5].

4.2. Palindromes and Lyndon words. A (weighted) alphabet is a set that is graded by the positive integers and whose homogeneous components are finite. The elements of degree $n$ are called letters of weight $n$. A word is a sequence of letters in the alphabet and a palindrome is a word that coincides with its reversal. The length of a word is the number of letters in the word, and the weight of a word is the sum of the weights of its letters.

Given an alphabet, let

$$
\operatorname{pal}(m) \text { and } \operatorname{npal}(m)
$$


denote the number of palindromes of weight $m$ and the number of nonpalindromic words of weight $m$. Also, let

$$
\operatorname{epal}(m) \text { and opal }(m)
$$

denote the number of palindromes of weight $m$ of even and odd length, and let

$$
\operatorname{pal}(k, m)
$$

denote the number of palindromes of length $k$ and weight $m$.

Assume now that a cofree graded connected Hopf algebra $H$ is given. Thus, $H \cong \mathcal{T}^{\vee}(V)$ as graded coalgebras, with $V=\mathcal{P}(H)$. Let

$$
v_{n}:=\operatorname{dim} V_{n}
$$

denote the dimension of the space of homogeneous primitive elements of degree $n$. Let $h_{n}$ and $g_{n}$ be as in Proposition 4, so $\left(h_{n}\right)$ is the dimension sequence of $H$ and $\left(g_{n}\right)$ is the dimension sequence of $\mathcal{P}(\tilde{H})$. Since $H \cong \mathcal{T}^{\vee}(V)$ as graded vector spaces, we have

$$
1+\sum_{m \geq 1} h_{m} t^{m}=\frac{1}{1-\sum_{n \geq 1} v_{n} t^{n}} .
$$

Together with (20), this yields

$$
1-\sum_{n \geq 1} v_{n} t^{n}=\prod_{i \geq 1}\left(1-t^{i}\right)^{g_{i}}
$$

In particular, the sequences $\left(h_{n}\right),\left(g_{n}\right)$, and $\left(v_{n}\right)$ determine each other.

Fix a homogeneous basis of $V$, and let it be our alphabet. Thus, there are $v_{n}$ letters of weight $n$. Equation (32) then says that $h_{m}$ is the total number of words of weight $m$. In particular,

$$
\operatorname{npal}(m)=h_{m}-\operatorname{epal}(m)-\operatorname{opal}(m) .
$$

Equation (33) says that $g_{i}$ is the number of Lyndon words of weight $i$ in the given alphabet. Then (16) says that mul $(k, m)$ counts the number of multisets of Lyndon words of cardinality $k$ and total weight $m$. Witt's formula [Kang and Kim 1996, Theorem 2.2] provides an explicit formula for $\left(g_{n}\right)$ in terms of $\left(v_{n}\right)$ :

$$
g_{n}=\sum_{d \mid n} \frac{\mu(d)}{d} \sum_{\lambda \vdash n / d} \frac{(\ell(\lambda)-1) !}{\lambda !} v^{\lambda},
$$

where $\mu(d)$ is the classical Möbius function, the inner sum is over all partitions $\lambda=1^{k_{1}} 2^{k_{2}} \cdots r^{k_{r}}$ of $n / d, \ell(\lambda)=k_{1}+k_{2}+\cdots+k_{r}, \lambda !=k_{1} ! k_{2} ! \cdots k_{r} !$, and

$$
v^{\lambda}:=v_{1}^{k_{1}} v_{2}^{k_{2}} \cdots v_{r}^{k_{r}} .
$$


(The special case of (34) in which all letters are of weight 1 appears in [Lothaire 1997, Corollary 5.3.5] and [Reutenauer 1993, Corollary 4.14].)

4.3. Characteristic polynomial and trace of the antipode in the cofree case. As an alternative to (19) and (24), we have the following expressions for the characteristic polynomial and the trace of the antipode of a cofree graded connected Hopf algebra $H$.

Theorem 21. For $H$ as above and any nonnegative integer $m$,

$$
\chi\left(\left.\mathrm{S}\right|_{H_{m}}\right)(x)=(x+1)^{\mathrm{opal}(m)}(x-1)^{\mathrm{epal}(m)}\left(x^{2}-1\right)^{\mathrm{npal}(m) / 2} .
$$

Proof. By Lemma 20, gr $H$ is isomorphic to the Hopf algebra $\mathcal{T}^{\vee}(V)$, where the latter is equipped with the shuffle product and the deconcatenation coproduct. As in Theorem 3, it suffices to analyze the antipode $\mathrm{S}$ of this Hopf algebra.

Now, it follows from (31) that each palindrome yields an eigenvector of $\mathrm{S}$. The eigenvalue is \pm 1 according to the parity of the length. This explains the first two factors in (35). The nonpalindromic words pair up with their reversals and organize in $2 \times 2$ blocks of the form

$$
\pm\left(\begin{array}{ll}
0 & 1 \\
1 & 0
\end{array}\right)
$$

where the sign again depends on the parity of the length. This accounts for the remaining factor.

As an immediate consequence, we have:

Corollary 22. For $H$ as above and any nonnegative integer $m$,

$$
\operatorname{trace}\left(\left.\mathrm{S}\right|_{H_{m}}\right)=\operatorname{epal}(m)-\operatorname{opal}(m)=\sum_{k=0}^{m}(-1)^{k} \operatorname{pal}(k, m) .
$$

Since there are no palindromes of even length and odd weight, (35) and (36) imply

$$
\chi\left(\left.\mathrm{S}\right|_{H_{m}}\right)(x)=(x+1)^{\mathrm{pal}(m)}\left(x^{2}-1\right)^{\mathrm{npal}(m) / 2}
$$

and

$$
\operatorname{trace}\left(\left.\mathrm{S}\right|_{H_{m}}\right)=-\operatorname{pal}(m)
$$

for all odd $m$.

Example 23. Let $V$ be an $r$-dimensional vector space. We view it as a graded vector space concentrated in degree 1 and consider the Hopf algebra $\mathcal{T}^{\vee}(V)$. Our alphabet consists of $r$ letters of weight 1 , and the palindrome distribution is

$$
\operatorname{pal}(k, m)= \begin{cases}r^{k} & \text { if } m=2 k \\ r^{k+1} & \text { if } m=2 k+1 \\ 0 & \text { otherwise. }\end{cases}
$$


It follows from (36) that

$$
\operatorname{trace}\left(\left.\mathrm{S}\right|_{H_{m}}\right)= \begin{cases}r^{m / 2} & \text { if } m \text { is even, } \\ -r^{(m+1) / 2} & \text { if } m \text { is odd. }\end{cases}
$$

We arrived at the same conclusion by different means in Example 14.

We return to the general discussion. From (24) and (36), we deduce

$$
\operatorname{epal}(m)-\operatorname{opal}(m)=\operatorname{emul}(m)-\operatorname{omul}(m),
$$

or equivalently,

$$
\sum_{k=0}^{m}(-1)^{k} \operatorname{pal}(k, m)=\sum_{k=0}^{m}(-1)^{k} \operatorname{mul}(k, m) .
$$

In general, the pairs (epal, opal) and (emul, omul), as well as the triangular arrays pal and mul, are different.

Example 24. Consider again the Malvenuto-Reutenauer Hopf algebra SSym. We compare the integers $\operatorname{mul}(k, m)$ and $\operatorname{pal}(k, m)$ for low values of $k$ and $m$.

Now, SSym is cofree and the relevant alphabet is the set of permutations with no global descents; see [Aguiar and Sottile 2005b, Corollary 6.3]. On the component of degree $m=3$, we have the following distribution of palindromes.

\begin{tabular}{l|ccc} 
length $(k)$ & 1 & 2 & 3 \\
\hline permutations & $123,132,213$ & 231,312 & 321 \\
words on alphabet & $123,132,213$ & $12|1,1| 12$ & $1|1| 1$ \\
\hline $\operatorname{pal}(k, 3)$ & 3 & 0 & 1
\end{tabular}

Beneath each permutation, we recorded its expression as words in the alphabet. Counting those words that are palindromic we obtained the integers $\operatorname{pal}(k, 3)$.

The integer $v_{m}$ is the number of permutations of $m$ elements with no global descents. The integers $\left(g_{m}\right)$ are calculated from either (20) or (33). The first few values are as follows.

\begin{tabular}{c|cccccc}
$m$ & 1 & 2 & 3 & 4 & 5 & 6 \\
\hline$v_{m}$ & 1 & 1 & 3 & 13 & 71 & 461 \\
$g_{m}$ & 1 & 1 & 4 & 17 & 92 & 572
\end{tabular}

The sequences $\left(v_{m}\right)$ and $\left(g_{m}\right)$ are A003319 and A112354 in [OEIS $\left.\geq 2015\right]$. Finally, the integers $\operatorname{mul}(k, m)$ are computed from (16). For $m=3$, we find the following:

\begin{tabular}{c|rrr}
$k$ & 1 & 2 & 3 \\
\hline $\operatorname{mul}(k, 3)$ & 4 & 1 & 1
\end{tabular}




\begin{tabular}{|c|c|c|c|c|c|c|c|c|c|c|c|c|c|}
\hline$k \backslash m$ & 1 & 2 & 3 & 4 & 5 & 6 & $k \backslash m$ & 1 & 2 & 3 & 4 & 5 & 6 \\
\hline 1 & 1 & 1 & 4 & 17 & 92 & 572 & 1 & 1 & 1 & 3 & 13 & 71 & 461 \\
\hline 2 & & 1 & 1 & 5 & 21 & 119 & 2 & & 1 & 0 & 1 & 0 & 3 \\
\hline 3 & & & 1 & 1 & 5 & 22 & 3 & & & 1 & 1 & 4 & 14 \\
\hline 4 & & & & 1 & 1 & 5 & 4 & & & & 1 & 0 & 2 \\
\hline 5 & & & & & 1 & 1 & 5 & & & & & 1 & 1 \\
\hline 6 & & & & & & 1 & 6 & & & & & & 1 \\
\hline
\end{tabular}

Figure 1. The arrays $\operatorname{mul}(k, m)$ (left) and pal $(k, m)$ (right) for $\mathfrak{S S y m}$.

Beyond $m=3$, the integers $\operatorname{mul}(k, m)$ and $\operatorname{pal}(k, m)$ differ more drastically; see Figure 1. However, the alternating sum of the entries in each column is the same for both arrays, as predicted by (38).

4.4. Generating functions. We continue to employ the notation of Section 4.2. Let

$$
v(t):=\sum_{n \geq 1} v_{n} t^{n}
$$

be the generating function for the dimension sequence of $V$.

We have the following generating functions for even and odd palindromes. The functions are bivariate to account for length and weight.

\section{Proposition 25.}

$$
\begin{aligned}
\sum_{k, m \geq 0} \operatorname{pal}(2 k, m) s^{k} t^{m} & =\frac{1}{1-s v\left(t^{2}\right)}, \\
\sum_{k, m \geq 0} \operatorname{pal}(2 k+1, m) s^{k} t^{m} & =\frac{v(t)}{1-s v\left(t^{2}\right)} .
\end{aligned}
$$

Proof. Consider a palindrome of even length $2 k$. Removing the first and last letters (which are equal) yields a palindrome of length $2 k-2$. The weights of the two palindromes differ by twice the weight of this letter. Therefore,

$$
\operatorname{pal}(2 k, m)=v_{1} \operatorname{pal}(2 k-2, m-2)+v_{2} \operatorname{pal}(2 k-2, m-4)+\cdots .
$$

This recursion leads at once to (39). A similar argument establishes (40).

Corollary 26.

$$
\sum_{m \geq 0} \operatorname{trace}\left(\left.\mathrm{S}\right|_{H_{m}}\right) t^{m}=\frac{1-v(t)}{1-v\left(t^{2}\right)} .
$$

Proof. This follows by subtracting (40) from (39), letting $s=1$, and using (36).

Remark. Corollary 26 is a special case of Corollary 13, in view of (32). The above may be regarded as a semicombinatorial proof of this result, which is possible under the cofreeness assumption. 


\section{Examples}

We carry out explicit calculations for the Hopf algebra of symmetric functions and a few related Hopf algebras, focusing on the trace of the antipode. They offer no difficulty, as explicit formulas for the antipodes of these Hopf algebras are known. Our purpose here is simply to illustrate some of the results from the preceding sections.

The paper [Aguiar et al. 2006] contains a concise description of each of the Hopf algebras discussed in this section. Other references are given as we proceed.

5.1. Symmetric functions. Consider the Hopf algebra of symmetric functions $H=$ Sym. See [Macdonald 1995, Chapter I] for the results used below. On the basis of Schur functions, the antipode acts by $S\left(s_{\lambda}\right)=(-1)^{|\lambda|} s_{\lambda^{\prime}}$, where $\lambda^{\prime}$ is the partition conjugate to $\lambda$. Therefore,

$$
\operatorname{trace}\left(\left.\mathrm{S}\right|_{H_{m}}\right)=(-1)^{m} c(m)
$$

where $c(m)$ is the number of self-conjugate partitions of $m$.

We turn to Corollary 10. For this Hopf algebra, $g_{i}=1$ for all $i \geq 1$. Hence $\left(\begin{array}{l}g \\ \lambda\end{array}\right)=1$ for all $\lambda$, and $\operatorname{mul}(k, m)=p_{k}(m)$, the number of partitions of $m$ into $k$ parts. From (24) we deduce

$$
(-1)^{m} c(m)=\sum_{k=0}^{m}(-1)^{k} p_{k}(m) .
$$

(Note that $p_{o}(m)=0$ for $m>0$.) The number of odd parts in a partition of $m$ has the same parity as $m$. Hence, the previous identity is equivalent to

$$
c(m)=e(m)-o(m),
$$

where $e(m)$ and $o(m)$ denote the number of partitions of $m$ with an even number of even parts and with an odd number of even parts. This identity appears in [Aigner 2007, Exercise 1.60] and [Stanley 2012, Chapter 1, Exercise 22(b)].

It is possible to obtain this result more directly as follows. Consider the power sum basis of Sym. Since $S\left(p_{\lambda}\right)=(-1)^{\ell(\lambda)} p_{\lambda}$, we have

$$
\operatorname{trace}\left(\left.\mathrm{S}\right|_{H_{m}}\right)=p_{e}(m)-p_{o}(m),
$$

where $p_{\boldsymbol{e}}(\mathrm{m})$ and $p_{o}(m)$ are the number of partitions of $m$ of even length and of odd length. Equating (42) and (45) gives (43) again.

We further illustrate Corollary 10 by deriving certain identities involving the Littlewood-Richardson coefficients $c_{\mu, \nu}^{\lambda}$. Recall that the latter are the structure constants for both the product and coproduct on the Schur basis of Sym,

$$
s_{\mu} \cdot s_{\nu}=\sum_{\lambda} c_{\mu, \nu}^{\lambda} s_{\lambda} \quad \text { and } \quad \Delta\left(s_{\lambda}\right)=\sum_{\mu, v} c_{\mu, \nu}^{\lambda} s_{\mu} \otimes s_{\nu}
$$


Formula (23) (with $n= \pm 2$ ) yields the following identities, for all $m \geq 1$ :

$$
\sum_{\lambda, \mu, \nu \vdash m}\left(c_{\mu, \nu}^{\lambda}\right)^{2}=\sum_{k=1}^{m} 2^{k} p_{k}(m)
$$

and

$$
\sum_{\lambda, \mu, \nu \vdash m} c_{\mu, \nu}^{\lambda} c_{\mu^{\prime}, v^{\prime}}^{\lambda}=\sum_{k=1}^{m}(-1)^{m-k} 2^{k} p_{k}(m) .
$$

Incidentally, the fact that the antipode preserves (co)products says that $c_{\mu, v}^{\lambda}=c_{\mu^{\prime}, v^{\prime}}^{\lambda^{\prime}}$

5.2. Schur $P$-functions. A partition of an integer is strict if its parts are all distinct. It is odd if each of its parts is odd.

Let $\lambda$ be a strict partition and $P_{\lambda} \in$ Sym the corresponding Schur $P$-function, as in [Macdonald 1995, Section III.8]. Let $H$ be the subspace of Sym spanned by the $P_{\lambda}$, as $\lambda$ runs over all strict partitions. Then $H$ is a Hopf subalgebra of Sym. We have

$$
\mathrm{S}\left(P_{\lambda}\right)=(-1)^{|\lambda|} P_{\lambda} \text {. }
$$

Therefore,

$$
\operatorname{trace}\left(\left.\mathrm{S}\right|_{H_{m}}\right)=(-1)^{m} p_{d}(m),
$$

where $p_{d}(m)$ is the number of strict partitions of $m$.

It is known that $H$ is the subalgebra of Sym generated by the odd power sums $p_{2 i+1}$ for $i \geq 0$. Therefore,

$$
\operatorname{trace}\left(\left.\mathrm{S}\right|_{H_{m}}\right)=(-1)^{m} p_{o}(m),
$$

where $p_{o}(m)$ is the number of odd partitions of $m$. Equating (48) and (49) recovers the classical fact that odd and strict partitions are equinumerous [Stanley 2012, Proposition 1.8.5].

Regarding the quantities in Proposition 4, we have that $g_{i}=1$ if $i$ is odd and 0 otherwise. It follows that $\left(\begin{array}{l}g \\ \lambda\end{array}\right)=1$ when $\lambda$ is odd and $\left(\begin{array}{l}g \\ \lambda\end{array}\right)=0$ otherwise. Therefore, $\operatorname{mul}(k, m)$ is the number of odd partitions of $m$ of length $k$. In an odd partition, the parities of $m$ and $k$ are the same. Thus, identity (24) simply counts odd partitions according to their length.

5.3. Quasisymmetric functions. Let us turn to the Hopf algebra $H=$ QSym of quasisymmetric functions. Consider the fundamental and monomial quasisymmetric functions, denoted by $F_{\alpha}$ and $M_{\alpha}$, respectively. As $\alpha$ runs over the compositions of $m$, both $\left\{F_{\alpha}\right\}$ and $\left\{M_{\alpha}\right\}$ constitute bases of $H_{m}$.

The antipode has the following descriptions:

$$
\mathrm{S}\left(F_{\alpha}\right)=(-1)^{m} F_{\tilde{\alpha}^{\prime}} \quad \text { and } \quad \mathrm{S}\left(M_{\alpha}\right)=(-1)^{\ell(\alpha)} \sum_{\beta \leq \alpha} M_{\tilde{\beta}}
$$


where $\tilde{\gamma}$ is the reversal of $\gamma, \gamma^{\prime}$ is its conjugate (obtained by reflecting the ribbon diagram of $\gamma$ across the main diagonal), and $\leq$ is the refinement partial order on compositions. Note that $\alpha=\tilde{\alpha}^{\prime}$ if and only if $\alpha$ is symmetric with respect to reflection across the antidiagonal. There are precisely $2^{(m-1) / 2}$ of these when $m$ is odd and zero when $m$ is even. Calculating the trace on the fundamental basis we thus obtain

$$
\operatorname{trace}\left(\left.\mathrm{S}\right|_{H_{m}}\right)= \begin{cases}-2^{(m-1) / 2} & \text { if } m \text { is odd } \\ 0 & \text { otherwise. }\end{cases}
$$

The compositions $\alpha$ that contribute to the trace on the monomial basis satisfy $\tilde{\alpha} \leq \alpha$. Since reversal is an order-preserving involution, this happens if and only if $\tilde{\alpha}=\alpha$, that is, when $\alpha$ is palindromic. Let $\operatorname{pal}(m)$ denote the number of palindromic compositions of $m$. If $m$ is even, exactly half of the palindromic compositions of $m$ have odd length; if $m$ is odd, all of them do. We conclude that

$$
\operatorname{trace}\left(\left.S\right|_{H_{m}}\right)= \begin{cases}-\operatorname{pal}(m) & \text { if } m \text { is odd, } \\ 0 & \text { otherwise }\end{cases}
$$

One may arrive at the same identity from (36).

Equating (50) and (51) we deduce that, for all odd $m$,

$$
\operatorname{pal}(m)=2^{(m-1) / 2} .
$$

It is easy to give a direct proof of this fact (and of pal $(m)=2^{\lfloor m / 2\rfloor}$ for all $m \geq 0$ ).

Since QSym is cofree, Theorem 21 applies. The space of primitive elements is spanned by the monomials $M_{(n)}$ for $n \geq 1$. One finds that

$$
\operatorname{pal}(k, m)=\left\{\begin{array}{cl}
\left(\begin{array}{c}
\lceil m / 2\rceil-1 \\
\lceil k / 2\rceil-1
\end{array}\right) & \text { if } m \text { is even, or if } m \text { is odd and } k \text { is odd, } \\
0 & \text { if } m \text { is odd and } k \text { is even. }
\end{array}\right.
$$

Formula (36) boils down in this case to the basic identities $2^{h}=\sum_{j=0}^{h}\left(\begin{array}{l}h \\ j\end{array}\right)$ and $0^{h}=\sum_{j=0}^{h}(-1)^{j}\left(\begin{array}{c}h \\ j\end{array}\right)$.

Regarding the quantities in Proposition 4, we have by (33) that $g_{i}$ is the number of Lyndon words of weight $i$ in an alphabet with one letter of weight $n$ for each $n \geq 1$. This number is given by $g_{1}=1$ and

$$
g_{i}=\frac{1}{i} \sum_{d \mid i} \mu(d) 2^{i / d}
$$

for $i \geq 2$ [Kang and Kim 1996, Proposition 2.3].

5.4. Peak quasisymmetric functions. Let $H$ be the Hopf algebra of peak quasisymmetric functions [Stembridge 1997]. It is a Hopf subalgebra of QSym, with a 
basis $\theta_{\alpha}$ indexed by compositions $\alpha$ into odd parts (odd compositions). The number of odd compositions of $m$ is the Fibonacci number $f_{m}$ (with $f_{0}=f_{1}=f_{2}=1$ ).

A formula for the antipode of $H$ is given in [Billera et al. 2003]:

$$
\mathrm{S}\left(\theta_{\alpha}\right)=(-1)^{m} \theta_{\tilde{\alpha}}
$$

It follows that

$$
\operatorname{trace}\left(\left.\mathrm{S}\right|_{H_{m}}\right)= \begin{cases}f_{m / 2} & \text { if } m \text { is even, } \\ -f_{\lceil m / 2\rceil+1} & \text { if } m \text { is odd }\end{cases}
$$

(as palindromic odd compositions of $m$ arise from odd compositions of $m / 2$ ). One may arrive at the same identity from (36).

As for QSym, $H$ is cofree. There is one primitive element of degree $n$ for each odd $n$. One finds that

$$
\operatorname{pal}(k, m)=\left\{\begin{array}{cl}
\left(\begin{array}{c}
(m+k) / 4-1 \\
(m-k) / 4
\end{array}\right) & \text { if } m \text { is even and } 4 \mid(m-k), \\
\left(\begin{array}{c}
(m+k-1) / 4\rfloor \\
\lfloor(m-k+1) / 4\rfloor
\end{array}\right) & \text { if } m \text { and } k \text { are odd, } \\
0 & \text { otherwise. }
\end{array}\right.
$$

Information about these numbers can be found in [OEIS $\geq 2015$, A046854 and A168561].

Formula (36) yields the following basic identities:

$$
f_{h}=\sum_{j=0}^{\lfloor h / 2\rfloor}\left(\begin{array}{c}
h-j-1 \\
j
\end{array}\right) \text { for } h \geq 0 \quad \text { and } \quad f_{h}=\sum_{j=0}^{h-2}\left(\begin{array}{c}
\left\lfloor\frac{h+j}{2}\right\rfloor-1 \\
\left\lfloor\frac{h-j}{2}\right\rfloor-1
\end{array}\right) \quad \text { for } h \geq 2
$$

\section{Appendix A. Hopf monoids in species}

The results from the earlier sections admit variants for Hopf monoids in species. We list the main ones in this section, along with indications for the proofs, which are similar to the ones for graded connected Hopf algebras.

This section assumes familiarity with the notion of Hopf monoid in species, as developed in [Aguiar and Mahajan 2010; 2013] and with the notation employed there. For the most part, the latter (shorter) reference suffices. The antipode is discussed in [Aguiar and Mahajan 2013, §5] and the Adams operators (convolution powers of the identity) appear in [loc. cit., §14.4].

All Hopf monoids $\boldsymbol{H}$ are assumed to be connected and finite-dimensional. That is, $\boldsymbol{H}[\varnothing]$ is one-dimensional, and for each finite set $I$, the vector space $\boldsymbol{H}[I]$ is finite-dimensional.

A.1. Characteristic polynomial. The starting point is the following result, whose proof is similar to that of [Aguiar and Sottile 2005a, Proposition 1.6]. 
Lemma 27. The Hopf monoid gr $\boldsymbol{H}$ (associated to the coradical filtration of $\boldsymbol{H}$ ) is commutative.

Let $h_{m}:=\operatorname{dim} \boldsymbol{H}[m]$, and let

$$
\boldsymbol{h}(t):=1+\sum_{m \geq 1} h_{m} \frac{t^{m}}{m !}
$$

denote the exponential generating function for the dimension sequence of $\boldsymbol{H}$.

Theorem 28. For every scalar $n$ and finite set I, the characteristic polynomial of the restriction $\left.\Psi_{n}\right|_{\boldsymbol{H}[I]}$ of the $n$-th Adams operator is of the form

$$
\chi\left(\left.\Psi_{n}\right|_{\boldsymbol{H}[I]}\right)(x)=\prod_{k=0}^{m}\left(x-n^{k}\right)^{\mathrm{xmul}(k, m)},
$$

where $m=|I|$. The nonnegative integers $\mathrm{xmul}(k, m)$ are independent of $n$ and are determined by the dimension sequence of $\boldsymbol{H}$, as follows:

$$
\sum_{k, m \geq 0} \operatorname{xmul}(k, m) s \frac{t^{m}}{m !}=\boldsymbol{h}(t)^{s} .
$$

Proof. As in the proof of Theorem 3, a combination of Lemma 27 with the PBW and CMM theorems (for Hopf monoids in species [Aguiar and Mahajan 2013, §15]) shows that we can assume that

$$
\boldsymbol{H}=\mathcal{S}(\boldsymbol{P})
$$

for a certain positive species $\boldsymbol{P}$. Here, $\mathcal{S}(\boldsymbol{P})$ is the free commutative monoid on $\boldsymbol{P}$ with its canonical Hopf monoid structure [loc. cit., §7].

Let $\boldsymbol{p}(t)$ be the exponential generating function for the dimension sequence of $\boldsymbol{P}$. Since $\mathcal{S}(\boldsymbol{P})=\boldsymbol{E} \circ \boldsymbol{P}$, where $\boldsymbol{E}$ is the exponential species, we have

$$
\boldsymbol{h}(t)=\exp (\boldsymbol{p}(t)) \quad \text { and } \quad \boldsymbol{h}(t)^{s}=\exp (s \boldsymbol{p}(t)) .
$$

On the other hand, a direct calculation of the Adams operators on $\mathcal{S}(\boldsymbol{P})$ shows that the characteristic polynomial is as in (53) and that the integers $\operatorname{xmul}(k, m)$ are determined by

$$
\sum_{k, m \geq 0} \operatorname{xmul}(k, m) s^{k} \frac{t^{m}}{m !}=\exp (s \boldsymbol{p}(t)) .
$$

The result follows. 
A.2. Trace of the antipode. Let

$$
\boldsymbol{a}(t):=1+\sum_{m \geq 1} \operatorname{trace}\left(\left.\mathrm{S}\right|_{\boldsymbol{H}[m]}\right) \frac{t^{m}}{m !}
$$

denote the exponential generating function for the trace of the antipode of a Hopf monoid $\boldsymbol{H}$. This is none other than the reciprocal of the exponential generating function for the dimension sequence.

Corollary 29.

$$
\boldsymbol{a}(t)=\frac{1}{\boldsymbol{h}(t)} .
$$

Proof. This follows from Theorem 28, taking $n=-1$ in (53) and $s=-1$ in (54).

Example 30. Let $\boldsymbol{H}=\boldsymbol{\Sigma}$ be the Hopf monoid of set compositions [Aguiar and Mahajan 2013, §11.1]. We have

$$
\boldsymbol{h}(t)=\frac{1}{2-\exp t} .
$$

Therefore, $\boldsymbol{a}(t)=2-\exp t=1-\sum_{m \geq 1} t^{m} / m$ !, and we obtain

$$
\operatorname{trace}\left(\left.\mathrm{S}\right|_{\boldsymbol{H}[m]}\right)=-1
$$

for all $m \geq 1$. This result can also be obtained by a direct calculation, starting from either of the expressions for the antipode of $\Sigma$ given in [loc. cit., Proposition 59 or Theorem 60].

For an extension of this result, assume that there is a positive species $\boldsymbol{P}$ such that

$$
\boldsymbol{H} \cong \boldsymbol{L} \circ \boldsymbol{P}
$$

as species, where $\boldsymbol{L}$ is the species of linear orders. Not every Hopf monoid $\boldsymbol{H}$ is of this form, but this is the case if $\boldsymbol{H}$ is free or cofree [loc. cit., §6]. In this situation, we have

$$
\operatorname{trace}\left(\left.\mathrm{S}\right|_{\boldsymbol{H}[m]}\right)=-\operatorname{dim} \boldsymbol{P}[m] .
$$

Note that if $\boldsymbol{P}=\boldsymbol{E}$, then $\boldsymbol{H} \cong \boldsymbol{\Sigma}$, and (57) recovers (56).

Example 31. Let $\boldsymbol{H}=\boldsymbol{\Pi}$ be the Hopf monoid of set partitions [Aguiar and Mahajan 2013, §9.3]. We have

$$
\boldsymbol{h}(t)=\exp (\exp t-1)
$$

Therefore,

$\boldsymbol{a}(t)=\exp (1-\exp t)=1-t+\frac{t^{3}}{3 !}+\frac{t^{4}}{4 !}-2 \frac{t^{5}}{5 !}-9 \frac{t^{6}}{6 !}-9 \frac{t^{7}}{7 !}+50 \frac{t^{8}}{8 !}+267 \frac{t^{9}}{9 !}+\cdots$

It follows that

$$
\operatorname{trace}\left(\left.S\right|_{\boldsymbol{H}[m]}\right)=\Pi_{e}(m)-\Pi_{o}(m),
$$


where $\Pi_{e}(m)$ and $\Pi_{o}(m)$ denote the number of set partitions of $[m]$ into an even and an odd number of blocks, respectively. This result can also be obtained by a direct calculation, starting from either of the expressions for the antipode of $\Pi$ given in [loc. cit., Theorem 33 or Proposition 35].

Remark. The Hopf monoid $\Pi$ is in many ways parallel to the Hopf algebra Sym of symmetric functions (Section 5.1). A consequence of the preceding calculation, however, is that $\Pi$ does not admit a linear basis that behaves under the antipode in the same manner as the Schur basis of Sym. More precisely, there is no basis $\left\{s_{\pi} \mid \pi \vdash[m]\right\}$ of the space $\Pi[m]$ with the property that

$$
\mathrm{S}\left(s_{\pi}\right)=(-1)^{m} s_{\pi^{\prime}}
$$

for some map $\pi \rightarrow \pi^{\prime}$ on the set of set partitions of $[m]$. Indeed, if this were the case, the sequence of antipode traces would alternate in sign.

For an extension of the calculation in Example 31, let $\boldsymbol{H}$ be a Hopf monoid and $\boldsymbol{P}$ a positive species such that

$$
H \cong E \circ P
$$

as species. (The first part of the proof of Theorem 28 shows that every connected Hopf monoid is of this form.) In this situation, an $\boldsymbol{H}$-structure on a finite set $\boldsymbol{I}$ is an assembly of $\boldsymbol{P}$-structures, in the sense of [Bergeron et al. 1998, §1.4]. Conversely, one may regard a $\boldsymbol{P}$-structure as a connected $\boldsymbol{H}$-structure. We then have

$$
\operatorname{trace}\left(\left.\mathrm{S}\right|_{\boldsymbol{H}[m]}\right)=h_{\boldsymbol{e}}(m)-h_{o}(m),
$$

where $h_{\boldsymbol{e}}(m)$ and $h_{\boldsymbol{o}}(m)$ denote the number of $\boldsymbol{H}$-structures with an even and odd number of connected components, respectively. If $\boldsymbol{P}=\boldsymbol{E}$, then (59) recovers (58).

The combination of (55) and (59) provide a semicombinatorial description for the reciprocal of any power series arising as the exponential generating function for the dimension sequence of a connected Hopf monoid in species.

\section{Appendix B. q-Hopf algebras}

Fix a scalar $q$. A $q$-Hopf algebra is a Hopf monoid in the lax braided monoidal category of graded vector spaces, with lax braiding $V \otimes W \rightarrow W \otimes V$ given by

$$
x \otimes y \mapsto q^{m n} y \otimes x,
$$

where $x \in V$ and $y \in W$ are homogeneous elements of degrees $m$ and $n$. If $q=1$, a $q$-Hopf algebra is just a graded Hopf algebra as in Section 1.6. For information on $q$-Hopf algebras, see [Aguiar and Mahajan 2010, §2.3].

In this section we discuss extensions of some of the main results from earlier sections to the context of connected $q$-Hopf algebras. 
B.1. Cofreeness for connected q-Hopf algebras. Let $V$ be a graded vector space. When $x \in V_{n}$, we write $|x|=n$. The deconcatenation coalgebra on $V$ (Section 4.1), endowed with the $q$-shuffle product, is a connected $q$-Hopf algebra. The $q$-shuffle product of two homogeneous tensors $x_{1} \cdots x_{i}$ and $x_{i+1} \cdots x_{k}$ is the following element of $\mathcal{T}^{\vee}(V)$ :

$$
\sum_{\sigma} q^{\operatorname{inv}_{x}(\sigma)} x_{\sigma^{-1}(1)} \cdots x_{\sigma^{-1}(k)}
$$

where the sum is over all permutations $\sigma \in S_{k}$ such that

$$
\sigma(1)<\cdots<\sigma(i), \quad \sigma(i+1)<\cdots<\sigma(k),
$$

and

$$
\operatorname{inv}_{x}(\sigma):=\sum_{\substack{a<b \\ \sigma(a)>\sigma(b)}}\left|x_{a}\right|\left|x_{b}\right|
$$

We denote the resulting $q$-Hopf algebra by $\mathcal{T}_{q}^{\vee}(V)$. The antipode is given by

$$
\mathrm{S}\left(x_{1} x_{2} \cdots x_{k}\right)=(-1)^{k} q^{\mathrm{inv} x}(k) x_{k} \cdots x_{2} x_{1},
$$

where

$$
\operatorname{inv}_{x}(k):=\sum_{a<b}\left|x_{a}\right|\left|x_{b}\right|
$$

The following is an extension of Lemma 20. The proof of the latter result given in [Aguiar and Sottile 2005b, Propositions 1.4 and 1.5] yields its extension as well.

Lemma 32. Let $H$ be a connected q-Hopf algebra that is cofree as a graded coalgebra. Then

$$
\operatorname{gr} H \cong \mathcal{T}_{q}^{\vee}(V)
$$

as q-Hopf algebras, where $V=\mathcal{P}(H)$.

B.2. Characteristic polynomial and trace of the antipode. Let $H$ be a connected $q$-Hopf algebra that is cofree as a graded coalgebra. We lay the groundwork for a description of the characteristic polynomial of such a Hopf algebra. Let a weighted alphabet be given, with $v_{n}$ letters of weight $n$, as in Section 4.2. The multiweight of a word is the sequence of letter weights. If the word has weight $m$, its multiweight is a composition of $m$, and we write $\alpha \vDash m$.

Given a composition $\alpha$, let

$$
\operatorname{pal}(\alpha) \text { and } \operatorname{npal}(\alpha)
$$


denote the number of palindromes and nonpalindromes, respectively, of multiweight $\alpha$. If $\alpha=\left(a_{1}, \ldots, a_{k}\right)$, then

$$
\operatorname{pal}(\alpha)=\left\{\begin{array}{ll}
\prod_{i=1}^{\lceil k / 2\rceil} v_{a_{i}} & \text { if } \alpha=\tilde{\alpha}, \\
0 & \text { otherwise, }
\end{array} \quad \text { and } \quad \operatorname{npal}(\alpha)=\left(\prod_{i=1}^{k} v_{a_{i}}\right)-\operatorname{pal}(\alpha) .\right.
$$

(Recall that $\tilde{\alpha}=\left(a_{k}, \ldots, a_{1}\right)$ denotes the reversal of $\alpha$.) Let $\ell(\alpha)$ denote the length $k$ of $\alpha$, and let

$$
\operatorname{inv}(\alpha):=\sum_{1 \leq i<j \leq k} a_{i} a_{j}
$$

Let $H$ be as above. Fix a homogeneous basis of $V=\mathcal{P}(H)$ and take it as our alphabet. Thus, $v_{n}=\operatorname{dim} V_{n}$.

Theorem 33. For each nonnegative integer $m$, the characteristic polynomial of the antipode is

$$
\chi\left(\left.\mathrm{S}\right|_{H_{m}}\right)(x)=\prod_{\alpha \models m}\left(x-(-1)^{\ell(\alpha)} q^{\operatorname{inv}(\alpha)}\right)^{\operatorname{pal}(\alpha)}\left(x^{2}-q^{2 \operatorname{inv}(\alpha)}\right)^{\mathrm{npal}(\alpha) / 2} .
$$

Proof. Lemma 32 allows us to assume that $H=\mathcal{T}_{q}^{\vee}(V)$. The result follows from (60), as in the proof of Theorem 21.

In particular, the eigenvalues of the antipode of such a $q$-Hopf algebra are positive or negative powers of $q$. We record the resulting expression for the trace.

Corollary 34.

$$
\operatorname{trace}\left(\left.\mathrm{S}\right|_{H_{m}}\right)=\sum_{\alpha \models m}(-1)^{\ell(\alpha)} \operatorname{pal}(\alpha) q^{\mathrm{inv}(\alpha)} .
$$

We recover (35) and (36) as the case $q=1$ of (61) and (62).

Example 35. Let $V$ be an $r$-dimensional vector space. View it as a graded vector space concentrated in degree 1 and consider the $q$-Hopf algebra $\mathcal{T}_{q}^{\vee}(V)$. Then

$$
\operatorname{pal}(\alpha)= \begin{cases}r^{\lceil m / 2\rceil} & \text { if } \alpha=\left(1^{m}\right) \\ 0 & \text { otherwise }\end{cases}
$$

Therefore,

$$
\operatorname{trace}\left(\left.\mathrm{S}\right|_{H_{m}}\right)=(-1)^{m} r^{\lceil m / 2\rceil} q^{\left(\begin{array}{c}
m \\
2
\end{array}\right)} .
$$

This generalizes the conclusion of Example 14.

B.3. Generating functions. We continue to assume that $H$ is a connected $q$-Hopf algebra that is cofree as a graded coalgebra, and $V=\mathcal{P}(H)$. We also assume that $q \neq 0$.

Let

$$
v_{q}(t):=\sum_{n \geq 1} v_{n} \frac{t^{n}}{q^{\left(\begin{array}{c}
n \\
2
\end{array}\right)}}
$$


All generating functions in this section will be of this form.

For each pair of nonnegative integers $k$ and $m$, let

$$
\operatorname{pal}_{q}(k, m):=\sum_{\substack{\alpha \in m \\ \ell(\alpha)=k}} \operatorname{pal}(\alpha) q^{\operatorname{inv}(\alpha)} .
$$

Then (62) may be rewritten as

$$
\operatorname{trace}\left(\left.\mathrm{S}\right|_{H_{m}}\right)=\sum_{k=0}^{m}(-1)^{k} \operatorname{pal}_{q}(k, m) .
$$

We have the following $q$-generating functions for even and odd palindromes, generalizing Proposition 25.

\section{Proposition 36.}

$$
\begin{aligned}
\sum_{k, m \geq 0} \operatorname{pal}_{q}(2 k, m) s^{k} \frac{t^{m}}{q^{\left(\begin{array}{c}
m \\
2
\end{array}\right)}} & =\frac{1}{1-s v_{q^{2}}\left(t^{2}\right)}, \\
\sum_{k, m \geq 0} \operatorname{pal}_{q}(2 k+1, m) s^{k} \frac{t^{m}}{q^{\left(\begin{array}{c}
m \\
2
\end{array}\right)}} & =\frac{v_{q}(t)}{1-s v_{q^{2}}\left(t^{2}\right)} .
\end{aligned}
$$

Proof. Consider a palindrome of even length $2 k$ and weight $m>0$. Its multiweight $\alpha$ is a palindromic composition of $m$, necessarily of the form

$$
\alpha=(a, \beta, a),
$$

where $a$ is a positive integer and $\beta$ is a palindromic composition of $m-2 a$. We have

$$
\operatorname{inv}(\alpha)=\operatorname{inv}(\beta)+a^{2}+2 a(m-2 a) \quad \text { and } \quad \operatorname{pal}(\alpha)=v_{a} \operatorname{pal}(\beta) .
$$

The former is equivalent to

$$
\operatorname{inv}(\alpha)-\left(\begin{array}{c}
m \\
2
\end{array}\right)=\operatorname{inv}(\beta)-\left(\begin{array}{c}
m-2 a \\
2
\end{array}\right)-2\left(\begin{array}{l}
a \\
2
\end{array}\right)
$$

Therefore,

$$
\begin{aligned}
\frac{\operatorname{pal}_{q}(2 k, m)}{q^{\left(\begin{array}{c}
m \\
2
\end{array}\right)}} & =\sum_{\substack{\alpha \models m \\
\ell(\alpha)=k}} \operatorname{pal}(\alpha) q^{\operatorname{inv}(\alpha)-\left(\begin{array}{c}
m \\
2
\end{array}\right)} \\
& =\sum_{a \geq 1} \sum_{\substack{\beta \models m-2 a \\
\ell(\beta)=2 k-2}} v_{a} \operatorname{pal}(\beta) q^{\operatorname{inv}(\beta)-\left(\begin{array}{c}
m-2 a \\
2
\end{array}\right)-2\left(\begin{array}{c}
a \\
2
\end{array}\right)} \\
& =\sum_{a \geq 1} \frac{v_{a}}{q^{2\left(\begin{array}{c}
a \\
2
\end{array}\right)}} \frac{\operatorname{pal}_{q}(2 k-2, m-2 a)}{q^{\left(\begin{array}{c}
m-2 a \\
2
\end{array}\right)}}
\end{aligned}
$$


This recursion leads to (64). A similar argument establishes (65).

We arrive at a generalization of Corollary 26:

Corollary 37.

$$
\sum_{m \geq 0} \operatorname{trace}\left(\left.\mathrm{S}\right|_{H_{m}}\right) \frac{t^{m}}{q^{\left(\begin{array}{c}
m \\
2
\end{array}\right)}}=\frac{1-v_{q}(t)}{1-v_{q^{2}}\left(t^{2}\right)} .
$$

Proof. This follows by subtracting (65) from (64), letting $s=1$, and using (63).

B.4. q-deformations. The results in the Sections B.2 and B.3 apply only under the assumption of cofreeness. For $q$-Hopf algebras, this hypothesis is less restrictive than it may seem, as we now argue.

Suppose our $q$-Hopf algebra is obtained by deforming the product of an ordinary Hopf algebra and leaving the unit and the coalgebra structure unchanged. Thus, we have a family of products $\mu_{q}$ on a coalgebra $H$, turning it into a connected $q$-Hopf algebra for each $q$, which we denote by $H(q)$. Assume also that $\mu_{q}$ depends polynomially on $q$. An example is $\mathcal{T}_{q}^{\vee}(V)$, which is a deformation of $\mathcal{T}^{\vee}(V)$. More generally, the $q$-Hopf algebras constructed from Hopf monoids in species by means of the functor $\mathcal{K}_{V, q}^{\vee}$, as in [Aguiar and Mahajan 2010, §19.7], are all of this form.

In this situation, we may consider the 0 -Hopf algebra $H(0)$. A result of Loday and Ronco [2006, Theorem 2.6] (see also [Aguiar and Mahajan 2010, Theorem 2.13]) guarantees that $H(0)$ is cofree as a graded coalgebra. Since the coproduct has not been deformed, we have that our $q$-Hopf algebra $H(q)$ is cofree for all $q$.

In particular, the preceding results apply to such Hopf algebra deformations. By duality, they also apply in situations where the coproduct has been polynomially deformed while the rest of the structure has been kept.

B.5. (-1)-Hopf algebras. The results of Section 2 relied on the PBW and CMM theorems for graded connected Hopf algebras. While these results are not available for general $q$-bialgebras, they are for $q= \pm 1$. In particular, the eigenvalues of the antipode of a (-1)-Hopf algebra are still \pm 1 , and the characteristic polynomials of the Adams operators take the form (19). (The multiplicities mul $(k, m)$ are no longer given by (16).)

\section{References}

[Aguiar and Lauve 2013] M. Aguiar and A. Lauve, "Antipode and convolution powers of the identity in graded connected Hopf algebras", pp. 1053-1064 in 25th International Conference on Formal Power Series and Algebraic Combinatorics (Paris, 2013), Assoc. Discrete Math. Theor. Comput. Sci., Nancy, 2013. MR 3091063 Zbl 1294.05165

[Aguiar and Mahajan 2010] M. Aguiar and S. Mahajan, Monoidal functors, species and Hopf algebras, CRM Monograph Series 29, Amer. Math. Soc., Providence, RI, 2010. MR 2012g:18009 Zbl 1209.18002 
[Aguiar and Mahajan 2013] M. Aguiar and S. Mahajan, "Hopf monoids in the category of species", pp. 17-124 in Hopf algebras and tensor categories, edited by N. Andruskiewitsch et al., Contemp. Math. 585, Amer. Math. Soc., Providence, RI, 2013. MR 3077234 Zbl 06342020

[Aguiar and Sottile 2005a] M. Aguiar and F. Sottile, "Cocommutative Hopf algebras of permutations and trees", J. Algebraic Combin. 22:4 (2005), 451-470. MR 2006g:16080 Zbl 1094.16024

[Aguiar and Sottile 2005b] M. Aguiar and F. Sottile, "Structure of the Malvenuto-Reutenauer Hopf algebra of permutations", Adv. Math. 191:2 (2005), 225-275. MR 2005m:05226 Zbl 1056.05139

[Aguiar et al. 2006] M. Aguiar, N. Bergeron, and F. Sottile, "Combinatorial Hopf algebras and generalized Dehn-Sommerville relations", Compos. Math. 142:1 (2006), 1-30. MR 2006h:05233 Zbl 1092.05070

[Aigner 2007] M. Aigner, A course in enumeration, Graduate Texts in Mathematics 238, Springer, Berlin, 2007. MR 2008f:05001 Zbl 1123.05001

[Bergeron et al. 1998] F. Bergeron, G. Labelle, and P. Leroux, Combinatorial species and tree-like structures, Encyclopedia of Mathematics and its Applications 67, Cambridge University Press, 1998. MR 2000a:05008 Zbl 0888.05001

[Billera et al. 2003] L. J. Billera, S. K. Hsiao, and S. van Willigenburg, "Peak quasisymmetric functions and Eulerian enumeration”, Adv. Math. 176:2 (2003), 248-276. MR 2005a:06003 Zbl 1027.05105

[Bonnafé and Hohlweg 2006] C. Bonnafé and C. Hohlweg, "Generalized descent algebra and construction of irreducible characters of hyperoctahedral groups", Ann. Inst. Fourier (Grenoble) 56:1 (2006), 131-181. MR 2007e:20015 Zbl 1098.20011

[Cartier 2007] P. Cartier, "A primer of Hopf algebras", pp. 537-615 in Frontiers in number theory, physics, and geometry, II, edited by P. M. Pierre Cartier, Bernard Julia and P. Vanhove, Springer, Berlin, 2007. MR 2008b:16059 Zbl 1184.16031

[Diaconis et al. 2014] P. Diaconis, C. Y. A. Pang, and A. Ram, "Hopf algebras and Markov chains: two examples and a theory", J. Algebraic Combin. 39:3 (2014), 527-585. MR 3183482 Zbl 1291.05220

[Flajolet and Sedgewick 2009] P. Flajolet and R. Sedgewick, Analytic combinatorics, Cambridge University Press, 2009. MR 2010h:05005 Zbl 1165.05001

[Gerstenhaber and Schack 1991] M. Gerstenhaber and S. D. Schack, "The shuffle bialgebra and the cohomology of commutative algebras", J. Pure Appl. Algebra 70:3 (1991), 263-272. MR 92e:13008 Zbl 0728.13003

[Kang and Kim 1996] S.-J. Kang and M.-H. Kim, "Free Lie algebras, generalized Witt formula, and the denominator identity”, J. Algebra 183:2 (1996), 560-594. MR 97e:17042 Zbl 0862.17001

[Kashina et al. 2002] Y. Kashina, G. Mason, and S. Montgomery, "Computing the Frobenius-Schur indicator for abelian extensions of Hopf algebras", J. Algebra 251:2 (2002), 888-913. MR 2003f:16061 Zbl 1012.16040

[Kashina et al. 2006] Y. Kashina, Y. Sommerhäuser, and Y. Zhu, "On higher Frobenius-Schur indicators", pp. viii+65 Mem. Amer. Math. Soc. 855, Amer. Math. Soc., Providence, RI, 2006. MR 2007k:16071 Zbl 1163.16029

[Kashina et al. 2012] Y. Kashina, S. Montgomery, and S.-H. Ng, "On the trace of the antipode and higher indicators”, Israel J. Math. 188 (2012), 57-89. MR 2897723 Zbl 1260.16027

[Kassel 1995] C. Kassel, Quantum groups, Graduate Texts in Mathematics 155, Springer, New York, 1995. MR 96e:17041 Zbl 0808.17003

[Linchenko and Montgomery 2000] V. Linchenko and S. Montgomery, "A Frobenius-Schur theorem for Hopf algebras", Algebr. Represent. Theory 3:4 (2000), 347-355. MR 2001k:16073 Zbl 0971.16018 
[Loday 1992] J.-L. Loday, Cyclic homology, Grundlehren der Mathematischen Wissenschaften [Fundamental Principles of Mathematical Sciences] 301, Springer, Berlin, 1992. MR 94a:19004 Zbl 0780.18009

[Loday and Ronco 2006] J.-L. Loday and M. Ronco, “On the structure of cofree Hopf algebras”, J. Reine Angew. Math. 592 (2006), 123-155. MR 2007b:16084 Zbl 1096.16019

[Lothaire 1997] M. Lothaire, Combinatorics on words, Cambridge University Press, 1997. MR 98g: $68134 \mathrm{Zbl} 0874.20040$

[Macdonald 1995] I. G. Macdonald, Symmetric functions and Hall polynomials, 2nd ed., Oxford University Press, New York, 1995. MR 96h:05207 Zbl 0824.05059

[Malvenuto and Reutenauer 1995] C. Malvenuto and C. Reutenauer, "Duality between quasisymmetric functions and the Solomon descent algebra”, J. Algebra 177:3 (1995), 967-982. MR 97d: 05277 Zbl 0838.05100

[Milnor and Moore 1965] J. W. Milnor and J. C. Moore, "On the structure of Hopf algebras", Ann. of Math. (2) 81 (1965), 211-264. MR 30 \#4259 Zbl 0163.28202

[Montgomery 1993] S. Montgomery, Hopf algebras and their actions on rings, CBMS Regional Conference Series in Mathematics 82, Amer. Math. Soc., Providence, RI, 1993. MR 94i:16019 Zbl 0793.16029

[Ng and Schauenburg 2008] S.-H. Ng and P. Schauenburg, "Central invariants and higher indicators for semisimple quasi-Hopf algebras", Trans. Amer. Math. Soc. 360:4 (2008), 1839-1860. MR 2009d:16065 Zbl 1141.16028

[Novelli et al. 2013] J.-C. Novelli, F. Patras, and J.-Y. Thibon, "Natural endomorphisms of quasishuffle Hopf algebras”, Bull. Soc. Math. France 141 (2013), 107-130. MR 3031674 Zbl 1266.05175

[OEIS] OEIS, "The On-Line Encyclopedia of Integer Sequences", http://oeis.org.

[Pang 2014] A. Pang, Hopf algebras and Markov chains, Ph.D. thesis, Stanford University, 2014, https://stacks.stanford.edu/file/druid:vy459jk2393/thesis_submit-augmented.pdf. Zbl 1291.05220

[Patras 1993] F. Patras, "La décomposition en poids des algèbres de Hopf”, Ann. Inst. Fourier (Grenoble) 43:4 (1993), 1067-1087. MR 95d:16054 Zbl 0795.16028

[Patras and Schocker 2006] F. Patras and M. Schocker, "Twisted descent algebras and the SolomonTits algebra”, Adv. Math. 199:1 (2006), 151-184. MR 2006k:16086 Zbl 1154.16029

[Quillen 1969] D. Quillen, "Rational homotopy theory", Ann. of Math. (2) 90 (1969), 205-295. MR 41 \#2678 Zbl 0191.53702

[Radford 2012] D. E. Radford, Hopf algebras, Series on Knots and Everything 49, World Scientific, Hackensack, NJ, 2012. MR 2894855 Zbl 1266.16036

[Reutenauer 1993] C. Reutenauer, Free Lie algebras, London Mathematical Society Monographs. New Series 7, The Clarendon Press, Oxford University Press, New York, 1993. MR 94j:17002 Zbl 0798.17001

[Sage and Vega 2012] D. S. Sage and M. D. Vega, "Twisted Frobenius-Schur indicators for Hopf algebras", J. Algebra 354 (2012), 136-147. MR 2012k:16087 Zbl 1280.16027

[Schmitt 1994] W. R. Schmitt, "Incidence Hopf algebras", J. Pure Appl. Algebra 96:3 (1994), 299330. MR 95m:16033 Zbl 0808.05101

[Serre 1977] J.-P. Serre, Linear representations of finite groups, Graduate Texts in Mathematics 42, Springer, New York, 1977. MR 56 \#8675 Zbl 0355.20006

[Shimizu 2012] K. Shimizu, "Some computations of Frobenius-Schur indicators of the regular representations of Hopf algebras", Algebr. Represent. Theory 15:2 (2012), 325-357. MR 2892511 Zbl 1260.16028 
[Sloane and Plouffe 1995] N. J. A. Sloane and S. Plouffe, The encyclopedia of integer sequences, Academic Press, San Diego, CA, 1995. MR 96a:11001 Zbl 0845.11001

[Stanley 2012] R. P. Stanley, Enumerative combinatorics, vol. 1, 2nd ed., Cambridge Studies in Advanced Mathematics 49, Cambridge University Press, 2012. MR 2868112 Zbl 1288.00026

[Stembridge 1997] J. R. Stembridge, "Enriched P-partitions", Trans. Amer. Math. Soc. 349:2 (1997), 763-788. MR 97f:06006 Zbl 0863.06005

[Sweedler 1969] M. E. Sweedler, Hopf algebras, W. A. Benjamin, New York, 1969. MR 40 \#5705 Zbl 0194.32901

[Taft 1971] E. J. Taft, “The order of the antipode of finite-dimensional Hopf algebra”, Proc. Nat. Acad. Sci. U.S.A. 68 (1971), 2631-2633. MR 44 \#4075 Zbl 0222.16012

[Takeuchi 1971] M. Takeuchi, "Free Hopf algebras generated by coalgebras", J. Math. Soc. Japan 23 (1971), 561-582. MR 45 \#1958 Zbl 0217.05902

[Wilf 2006] H. S. Wilf, generatingfunctionology, 3rd ed., A K Peters, Wellesley, MA, 2006. MR 2006i:05014 Zbl 1092.05001

Communicated by Susan Montgomery

Received 2014-03-27 Revised 2014-10-23 Accepted 2014-12-01

maguiar@math.cornell.edu Department of Mathematics, Cornell University, Ithaca, NY 14853, United States

lauve@math.luc.edu

Department of Mathematics and Statistics, Loyola University Chicago, Chicago, IL 60660, United States 


\section{Algebra \& Number Theory}

msp.org/ant

\section{EDITORS}

MANAGING EDITOR

Bjorn Poonen

Massachusetts Institute of Technology

Cambridge, USA

\author{
EDITORIAL BOARD CHAIR \\ David Eisenbud \\ University of California \\ Berkeley, USA
}

\section{BOARD OF EDITORS}

Georgia Benkart

Dave Benson

Richard E. Borcherds

John H. Coates

J-L. Colliot-Thélène

Brian D. Conrad

Hélène Esnault

Hubert Flenner

Edward Frenkel

Andrew Granville

Joseph Gubeladze

Roger Heath-Brown

Craig Huneke

János Kollár

Yuri Manin

Barry Mazur

Philippe Michel
University of Wisconsin, Madison, USA

University of Aberdeen, Scotland

University of California, Berkeley, USA

University of Cambridge, UK

CNRS, Université Paris-Sud, France

University of Michigan, USA

Freie Universität Berlin, Germany

Ruhr-Universität, Germany

University of California, Berkeley, USA

Université de Montréal, Canada

San Francisco State University, USA

Oxford University, UK

University of Virginia, USA

Princeton University, USA

Northwestern University, USA

Harvard University, USA

École Polytechnique Fédérale de Lausanne
Susan Montgomery

Shigefumi Mori

Raman Parimala

Jonathan Pila

Anand Pillay

Victor Reiner

Peter Sarnak

Joseph H. Silverman

Michael Singer

Vasudevan Srinivas

J. Toby Stafford

Ravi Vakil

Michel van den Bergh

Marie-France Vignéras

Kei-Ichi Watanabe

Efim Zelmanov

Shou-Wu Zhang
University of Southern California, USA

RIMS, Kyoto University, Japan

Emory University, USA

University of Oxford, UK

University of Notre Dame, USA

University of Minnesota, USA

Princeton University, USA

Brown University, USA

North Carolina State University, USA

Tata Inst. of Fund. Research, India

University of Michigan, USA

Stanford University, USA

Hasselt University, Belgium

Université Paris VII, France

Nihon University, Japan

University of California, San Diego, USA

Princeton University, USA

\section{PRODUCTION}

production@msp.org

Silvio Levy, Scientific Editor

See inside back cover or msp.org/ant for submission instructions.

The subscription price for 2015 is US $\$ 255 /$ year for the electronic version, and $\$ 440 /$ year ( $+\$ 55$, if shipping outside the US) for print and electronic. Subscriptions, requests for back issues and changes of subscribers address should be sent to MSP.

Algebra \& Number Theory (ISSN 1944-7833 electronic, 1937-0652 printed) at Mathematical Sciences Publishers, 798 Evans Hall \#3840, c/o University of California, Berkeley, CA 94720-3840 is published continuously online. Periodical rate postage paid at Berkeley, CA 94704, and additional mailing offices.

ANT peer review and production are managed by EditFLOW ${ }^{\circledR}$ from MSP.

\section{PUBLISHED BY}

- mathematical sciences publishers

nonprofit scientific publishing

http://msp.org/

(C) 2015 Mathematical Sciences Publishers 


\section{Algebra \& Number Theory}

Volume $9 \quad$ No. $3 \quad 2015$

Hurwitz monodromy and full number fields

DAVID P. ROBERTS and AKSHAY VENKATESH

The characteristic polynomial of the Adams operators on graded connected Hopf algebras

Marcelo Aguiar and Aaron Lauve

Secant spaces and syzygies of special line bundles on curves

MARIAN APRODU and EDOARDO SERNESI

Complex group algebras of the double covers of the symmetric and alternating groups

601

Christine Bessenrodt, Hung Ngoc Nguyen, Jørn B. Olsson and Hung P. TONG-VIET

Fano schemes of determinants and permanents

Melody Chan and NATHan Ilten

Triple intersection formulas for isotropic Grassmannians

VIJAY RAVIKUMAR

On the basepoint-free theorem for log canonical threefolds over the algebraic closure of a finite field

Diletta Martinelli, Yusuke NaKamura and Jakub Witaszek

The torsion group of endotrivial modules

JON F. CARLSON and JACQUES THÉVENAZ 